\title{
Joint Downlink/Uplink RF Wake-up Solution for IoT over Cellular Networks
}

\author{
Nour Kouzayha, Student Member, IEEE, Zaher Dawy, Senior Member, IEEE, Jeffrey G. Andrews, Fellow, IEEE, \\ and Hesham ElSawy, Senior Member, IEEE,
}

\begin{abstract}
We use stochastic geometry to analyze the performance of an energy-efficient joint downlink/uplink (DL/UL) radio frequency (RF) wake-up solution for Internet of Things (IoT) devices over cellular networks. When the IoT device has no data to transmit, it turns its main circuitry completely off and switches to a deep sleep mode. The transition back to the active mode is only achieved upon receiving enough power at the device's front-end. After wake-up, the device initiates regular UL communication with its serving base station (BS). The device experiences a successful wake-up event when the total received power includes a wake-up signal transmitted from its serving $B S$ and the UL signal-to-interference-and-noise ratio (SINR) is above a predefined threshold. On the other hand, the device experiences a false wake-up event when the wake-up is due to received power from neighboring BSs excluding the serving BS. We derive lower and upper bounds for the success and false wake-up probabilities in addition to closed-form expression for the UL SINR coverage probability after successful wake-up. We present performance results as a function of various key design parameters, and highlight the effectiveness and trade-offs of RF wake-up for IoT devices.
\end{abstract}

Index Terms-Internet of Things, RF wake-up, power consumption, stochastic geometry, energy efficiency.

\section{INTRODUCTION}

$\mathbf{E}$ VOLUTION towards smarter and more connected communities is being enabled by the emerging paradigm of the Internet of Things (IoT) where a huge number of devices will be connected wirelessly over diverse technologies that include both local area and cellular networks [1]. A founding pillar of IoT is the availability of devices with low-power wireless communication capabilities. These devices are usually powered by batteries with restricted size and capacity and are often deployed in locations that can be remote and not easily reachable. This has led to increasing interest in developing new energy efficient communication solutions for cellular-based IoT networks [2], [3], including novel resource allocation schemes [4], [5], discontinuous transmission mechanisms [6][8], censoring-based solutions [9]-[11], in addition to energy harvesting and RF wake-up solutions [12]-[15].

N. Kouzayha and Z. Dawy are with the Electrical and Computer Engineering Department, American University of Beirut, Lebanon. Email: nhk16@aub.edu.lb,zd03@aub.edu.lb.

J. G. Andrews is with the Wireless Networking and Communication Group, The University of Texas at Austin, Austin TX, USA. Email: jandrews@ece.utexas.edu.

H. ElSawy is with King Abdullah University of Science and Technology (KAUST), Saudi Arabia. Email: hesham.elsawy@kaust.edu.sa

Manuscript received xxxx, xxxx; revised xxxx, xxxx.

\section{A. Related Work}

RF wake-up is considered one of the key solutions for ultra-low power consumption [16]. The device enters the sleep mode to save power whenever it is not performing a useful task and only leaves the sleep mode when it receives an external RF wake-up signal. The on-demand characteristic of the RF wake-up solution ensures the high responsiveness of the device when requested for communication and reduces the idle listening activity, which consumes a notable portion of the battery energy. On-demand wake-up schemes are particularly suitable for IoT applications with low duty cycle (e.g., fire detection, surveillance of machine failures and, more generally, all event-driven scenarios). However, these solutions have not been considered comprehensively yet for enhancing the power efficiency of IoT over cellular; they have been widely used in wireless sensor network (WSN) and wireless local area network (WLAN) applications, with notable research advancements in hardware design for RF wake-up receivers [17]-[19].

Given the large-scale nature of IoT networks, stochastic geometry tools are particularly well suited for their analysis where both the devices and the BSs can be modeled as Point processes. These tools have already been used to model power efficient schemes in cellular networks, e.g., see [20][23]. For instance, [21] uses stochastic geometry to evaluate the probability of uplink transmission defined as the event of harvesting enough energy to transmit data to the nearest BS. The same performance metric is studied for a slightly different spatial setup in [22], where spatial repulsion between the locations of the RF sources is captured by modeling them as a realization of the Ginibre determinantal point process. In [23], the authors develop an energy aware traffic offloading scheme for heterogeneous cellular networks when the small cells are powered by grid power and energy harvesting while always satisfying the users requirements.

Motivated by the fact that stochastic geometry enables modeling and analysis of random spatial patterns, several authors have recently applied it to study the achievable performance of wireless powered communications (WPC) for large scale cellular networks [24]-[29]. WPC is an emerging concept where RF signals are used for information transmission and for powering-up mobile devices. The work in [24] explores power splitting receiver architecture for information and energy in a point-to-point system to study the trade-off between harvested energy and rate. In [27], the authors study outage and average energy harvested under power splitting using tools 
from stochastic geometry where the information and the power are transferred through a relay to the receiver. The authors in [28] explore power control policies for outage minimization in a point-to-point link assuming energy harvesting at both the transmitter and the receiver. The work in [29] relies on modeling the locations of BSs as points of a spatial Poisson point process to analyze the trade-offs emerging from simultaneous wireless information and power transfer transmission, while encompassing a realistic channel model that accounts for line of sight and non line of sight links, different cell association criteria, practical receivers based on time switching and power splitting schemes and directional beamforming.

In this work, we present an analytical framework to evaluate and optimize the performance of RF wake-up strategies for IoT devices over cellular networks. The power of the received wake-up signal is used to activate the circuitry of the device. Cellular networks, in fact are based on the assumption that the other-cell interference has a negative impact on the performance, since it reduces the coverage probability and average rate and increases the error probability. Interference, on the other hand, may be a natural source to be exploited for RF wake-up. Therefore, the device can benefit from the ambient interference of concurrent downlink transmissions to increase the amount of power received and the chance of having a successful wake-up. Note that a device can wake-up upon receiving sufficient amount of power from ambient interference without receiving a wake-up signal from its serving BS. We refer to this as a false wake-up event.

\section{B. Contribution and Organization}

The contributions of this paper can be summarized as follows:

- We propose the utilization of RF wake-up solutions for suitable IoT use cases over cellular networks in order to reduce the devices' energy consumption and enhance network efficiency. By adding a simple wake-up receiver at the front-end of the IoT device, the power of a specific wake-up signal transmitted from the serving BS along with the ambient interference can be captured and used to activate the device and initiate uplink communication.

- We provide a tractable framework for statistical analysis of the RF wake-up solution in cellular IoT networks. We derive expressions for the wake-up probability, as well as lower and upper bounds for the success wakeup probability and the false wake-up probability for different channel models. Under full channel inversion power control, we derive a closed-form expression for the SINR uplink coverage probability for the devices that have successfully woken-up and initiated communication.

- Via numerical results, we demonstrate the reliability and energy efficiency of RF wake-up solutions for IoT over cellular. In addition, we highlight the different trade-offs in the system and quantify the effect of various network parameters such as density of BSs and efficiency of the added wake-up receiver on the system performance.

Note that this work builds on our previous work [30] where we have analyzed the probabilities of the success and the false wake-up events assuming just a DL scenario. In this work, we extend the model such that, after successful wakeup, the device initiates uplink communication and sends data to its serving BS. Furthermore, we consider different channel models (pathloss only and with fading) while deriving bounds for the false wake-up probability. The rest of the paper is organized as follows: Section $[\mathrm{II}$ presents a general design for RF wake-up for IoT over cellular. Section III describes the system model including the network model, channel model, association criteria and the methodology of analysis. Section IV presents the derivations related to the wake-up probability. In Section V, lower and upper bounds on the false wake-up probability are derived, and in Section VI the success wakeup probability is derived. Section VII presents the analysis of the uplink SINR coverage probability, and Section VIII presents analysis on the energy efficiency of the proposed solution. Numerical results are discussed in Section IX with validation using Monte-Carlo simulations. Conclusions are drawn in Section $\mathrm{X}$.

Notation: Throughout the paper $\mathbb{P}\{\cdot\}$ denotes probability, $\mathbb{E}[\cdot]$ denotes expectation, $\mathcal{L}_{x}(\cdot)$ denotes the Laplace transform of a random variable $x, \triangleq$ denotes the definition, $\backsim$ denotes the distribution, $\operatorname{Re}(\cdot)$ denotes the real part of a complex number, $b(a, b)$ denotes a disc of radius $b$ centered at $a, \mathrm{Q}(x)=$ $\frac{1}{\sqrt{2 \pi}} \int_{x}^{\infty} e^{-\frac{t^{2}}{2}} \mathrm{dt}$ denotes the Q-function, ${ }_{2} F_{1}(a, b ; c ; x)=$ $\sum_{n=0}^{\infty} \frac{(a)_{n}(b)_{n}}{(c)_{n}} \frac{x^{n}}{n !}$ denotes the Gauss hypergeometric function, and $\Gamma[s, x]=\int_{x}^{\infty} t^{s-1} e^{-t} \mathrm{dt}$ denotes the incomplete gamma function.

\section{RF WAKe-Up SOlution FOR IOT OVER CELlulaR}

In the RF wake-up proposed solution for IoT devices, we define a deep sleep state similar to the power saving mode (PSM) defined by 3GPP [31]. A device that supports PSM will request the network for a certain active timer value; the active timer determines how long the device remains active, before it moves to PSM. In PSM, the device becomes unreachable as it does not check for paging but it is still registered with the network. The device remains in PSM until a mobileoriginated transaction requires it to initiate communication. Otherwise, the device leaves the PSM when a timer expires. The difference between our solution and the 3GPP model is that the device does not leave the deep sleep state when a timer expires but instead upon receiving an explicit RF wakeup signal from its serving BS. Fig. 1 presents the device states and the transactions defined in 3GPP along with the added deep sleep mode. The transition between the idle and the deep sleep modes is realized through an RF signal from the BS. A new added wake-up hardware module is responsible for capturing the wake-up signal and initiating an interrupt trigger to activate the main circuitry of the device. In order to improve the power efficiency, the power consumption of this extra wake-up radio receiver should be extremely low. A scheme representing a device incorporating the added wakeup module is presented in Fig. 2. The new wake-up module contains three main components: the matching network, a rectifier and an optional ID detector. The crucial task of the matching network is to reduce the transmission loss from the 


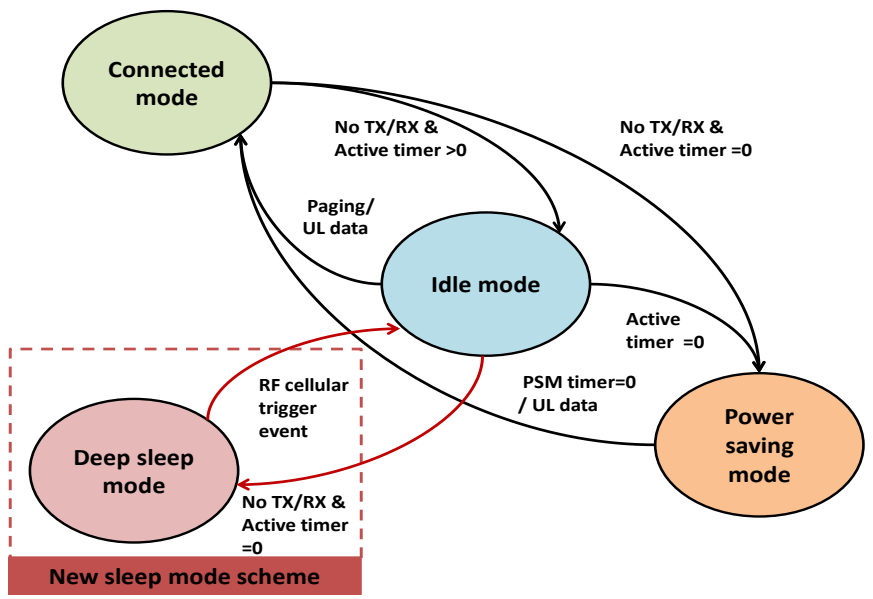

Fig. 1. Proposed RF wake-up IoT device states.

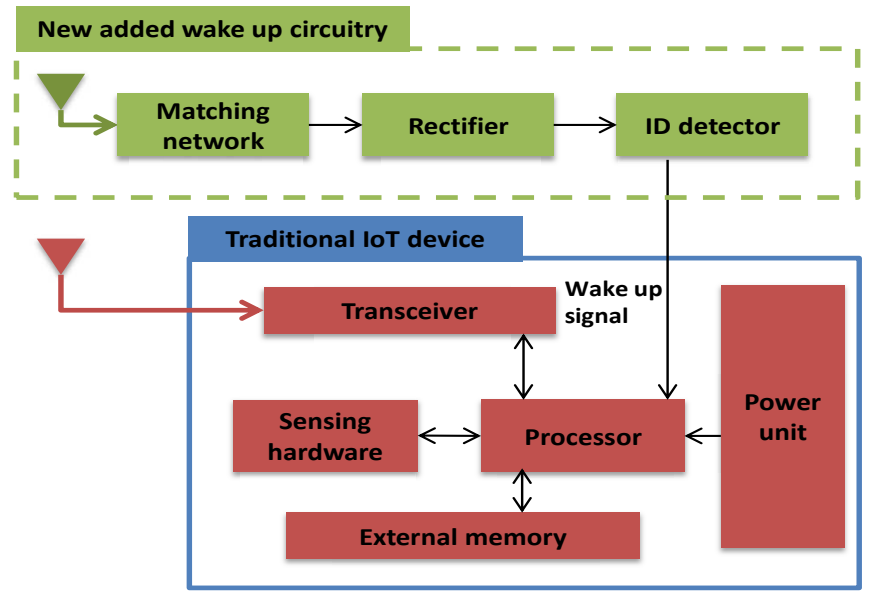

Fig. 2. IoT device employing the RF wake-up circuitry.

antenna to the rectifier circuit and to increase the input voltage of the rectifier. The rectifier is added to convert the RF-signals captured by the antenna into DC-voltage. The ID detector is responsible of extracting the ID of the received RF-signal and comparing it with the device's ID. In case of matched IDs, it will trigger the interrupt signal to activate the main circuitry of the device and transit to the idle mode. In the remaining of this work, we assume a broadcast wake-up scenario where all devices within the range of the transmitted wake-up signal will wake-up upon detecting enough signal power.

The proposed wake-up procedure is considered as an RF paging procedure. The main difference with the traditional paging procedure resides in the nature of the wake-up signal. While paging requests are exchanged through standard protocol messages and require customized detection schemes [32], the proposed solution benefits from the power of the RF wakeup signal to interrupt the device and switch it to the active state. To benefit from the advantages of universal frequency reuse in terms of spectral efficiency and channel allocation flexibility, we consider a scenario where all BSs transmit their wake-up signals on the same resource elements. Thus, there

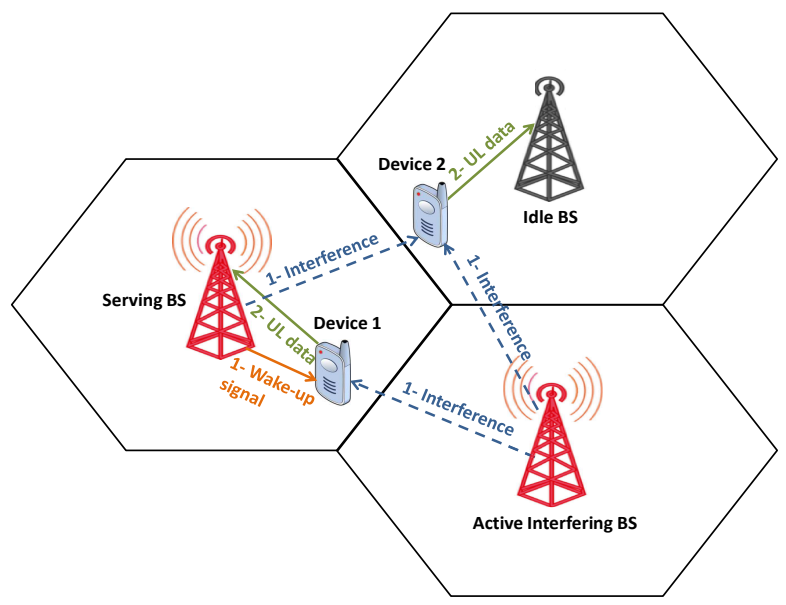

Fig. 3. Visual example model of the DL and UL events of the proposed RF wake-up procedure.

are no intra-cell interference problems. However, neighboring active BSs sending wake-up signals simultaneously can still affect the wake-up procedure. We define a power threshold $T$ such that a device wakes-up only when the total power received exceeds $T$. The efficiency of the wake-up module is modeled with an RF-DC conversion factor $a$ defined as the ratio between the power received at the input and the power at the output of the wake-up module.

The device can receive wake-up signals from neighboring BSs sending wake-up signals using the same resources. Such scenario can have a positive effect on the wake-up procedure as it increases the power received and, thus, the chance of having successful wake-up. However, if the device is not supposed to wake-up, receiving power from neighboring BSs may cause undesirable wake-ups; we denote this as a false wake-up event and we derive the false wake-up probability is Section $\mathrm{V}$. The complete wake-up procedure includes two successive events. First, the device should receive enough power given that its serving BS is transmitting the wake-up signal in the DL. The probability of this event is the success wake-up probability which will be derived in Section VI. Next, the device that has successfully woken-up will initiate communication with its serving BS in the UL and the level of the received SINR at the serving BS should exceed a predefined threshold, this uplink SINR coverage probability is derived in Section VII Fig. 3 presents an example model of the proposed RF wake-up solution where Device 1 encounters a success wake-up event and transmits UL data and Device 2 encounters a false wakeup event.

\section{System Model And Assumptions}

\section{A. Cellular Network Model}

The network model consists of BSs arranged according to an homogeneous Poisson point process (PPP) $\Phi=\left\{x_{i}, i=\right.$ $1,2, \ldots\} \in \mathbb{R}^{2}$ with spatial density $\lambda$ in the Euclidean plane. Usually, cellular networks are deployed according to sophisticated network planning processes, which would impose some repulsion between the BSs locations. Hence, it is intuitive 
TABLE I

NOTATIONS OF THE MAIN PARAMETERS USED IN THE ANALYSIS

\begin{tabular}{|l|l|}
\hline Symbol & Description \\
\hline$\phi, \lambda$ & Poisson point process, density of the set of BSs \\
\hline$\phi_{u}, \lambda_{u}$ & Poisson point process, density of the set of devices \\
\hline$a$ & RF-DC conversion factor \\
\hline$T, \tau$ & Power threshold in DL, SINR threshold in UL \\
\hline$P$ & Transmit power of a BS in the DL \\
\hline$\alpha$ & Path loss exponent \\
\hline$h$ & Fading channel gain \\
\hline$\delta$ & Active probability of BSs \\
\hline$\psi$ & Poisson point process of the set of active BSs \\
\hline$\Delta$ & Poisson point process of the set of idle BSs \\
\hline$\rho$ & Power level requirement of a BS in the UL \\
\hline$\gamma$ & Transmit power of a device in the UL \\
\hline$\tilde{\psi}$ & Poisson point process of the set of interfering devices in UL \\
\hline$P_{\text {main_ON }}, P_{\text {main_sleep }}$ & Power consumption of the main circuit in the active and sleep modes \\
\hline$P_{\text {Wur_ON }}, P_{\text {Wur_sleep }}$ & Power consumption of the wake-up receiver in the active and sleep modes \\
\hline$D_{\text {ON }}$ & Percentage of active time \\
\hline
\end{tabular}

that a repulsive point process is a better model for the BSs locations. It has been shown in $[33]-[35]$ that the homogeneous PPP captures the same shape (i.e., behavior) of the signal-to-interference-and-noise ratio (SINR) distribution as other repulsive point processes but with a horizontal shift. Hence, the homogeneous PPP represents the widely accepted baseline model for cellular networks, which can also be extended to repulsive point processes [36], [37]. The devices are spatially distributed in $\mathbb{R}^{2}$ according to an independent $\operatorname{PPP} \Phi_{u}=\left\{y_{i}, i=1,2, \ldots\right\}$ with spatial density $\lambda_{u}$. Each device is equipped with the wake-up module detailed in Section II with RF-DC conversion efficiency $a \leq 1$. We assume that all BSs are transmitting with the same power $P$ in the DL. The case of different BSs power levels corresponds to a multi-tier network where tiers of BSs differ in terms of the transmit power, deployment density, and target power threshold $T$. The multi-tier scenario is not considered in this work and is left for future extension. For the reliability of uplink transmission, and to mitigate the inter-cell interference, the devices use full channel inversion power control to adjust their transmission powers such that the average received power by the serving BS is constant and equal to the power level requirement $\rho$. Such power control implies that each BS is receiving the intended signal with power $\rho$, which is greater than the power of any individual interfering signal. It is also shown in [38] that full inversion power control minimizes the discrepancies of the coverage probability across the devices in the network. Without loss of generality, we focus on a device at the origin which is permissible by Slivnyak's theorem [39]. The remaining parameters used in the analysis are defined in Table []

\section{B. Channel Model and Association Criteria}

Channels are assumed to be symmetric where the power of a signal transmitted by any network node (BS or device) is subject to the standard power loss propagation model with rate $r^{-\alpha}$ where $\alpha>2$ is the pathloss exponent and $r$ is the propagation distance. In addition, we assume that the BSs and the devices experience independent Rayleigh fading and the

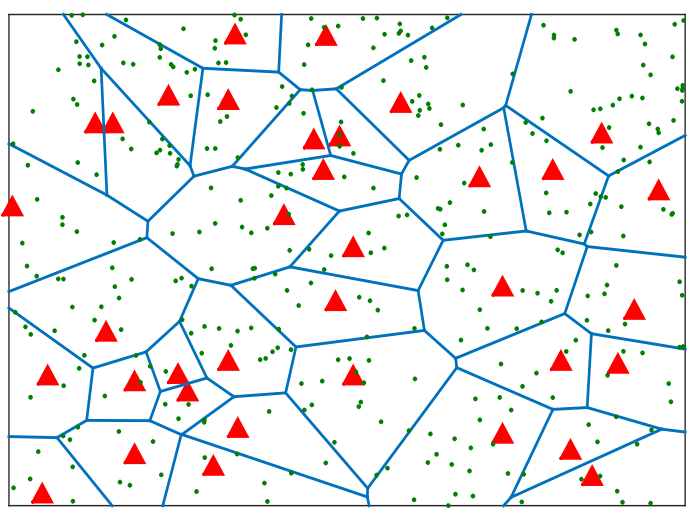

Fig. 4. Poisson distributed BSs (triangles) and devices (dots). The cell boundaries are shown and form a Voronoi tessellation.

corresponding channel power gain $h$ follow an exponential distribution with unit mean, i.e., $h \backsim \exp (1)$. Thus, the received power in DL at a device located at $y=\left(y_{1}, y_{2}\right)$ from its $\mathrm{BS}$ at $x=\left(x_{1}, x_{2}\right)$ is $P h\|x-y\|^{-\alpha}$ where $\|$. is the Euclidean norm. It is assumed that the DL and the UL transmissions are separated from each other in time or frequency such that there is no interference between them. We also assume that not all the BSs are active at the same time. The BSs which are active simultaneously and transmit on the same resources, are a thinned version of the PPP of BSs denoted as $\Psi$ with spatial density $\delta \lambda$, where $\delta$ is the probability that the BS is active. The remaining idle BSs constitute another PPP denoted as $\Delta$ with spatial density $(1-\delta) \lambda$. We assume that each device is associated with the closest $\mathrm{BS}$ to receive the wake-up signal in the downlink and transmit data in the uplink. This results in the creation of coverage areas that compromise a Voronoi tessellation on the plane. Fig. 4 shows a realization of the proposed network model.

\section{Methodology of Analysis}

Based on the system model described above, we aim at quantifying the performance of the proposed RF wake-up 
solution in terms of the following.

1) The success wake-up probability: The probability that the power received at the device given its serving $\mathrm{BS}$ is transmitting is sufficient to wake-up the device.

2) The false wake-up probability: The probability that the RF power collected from ambient is sufficient to wakeup the device, even though the serving BS is idle.

3) The SINR uplink coverage probability: The probability that the received SINR at the BS from the device after wake-up is above a predefined threshold.

To derive the success and the false wake-up probabilities, we first derive the Laplace transform of the DL received power from all active BSs and use it to derive the wake-up probability, which is the probability of achieving the power threshold $T$ regardless of whether or not the wake-up signal is transmitted. The DL received power is either the interference power itself in case the serving BS is idle or the interference plus the wake-up signal together if the serving BS is active. Thus, we obtain two expressions for the Laplace transform of the received power and we use them to obtain bounds for the false and the success probabilities. To obtain the SINR uplink coverage probability, we assume that the active devices after wake-up are now transmitting UL data to their serving BSs. We characterize first the UL transmit power of a generic device by deriving its probability density function (PDF) and moments. We use it then to derive the uplink SINR coverage probability. At the end, we demonstrate the efficiency of the proposed RF wake-up solution in terms of energy reduction by comparing it with the "scheduled wake-up" scheme.

\section{WAKE-UP PROBABILITY DERIVATION}

In order to wake-up, the device should receive a power $P_{r}$ greater than a threshold $T$. If the serving $\mathrm{BS}$ is active and transmitting the wake-up signal, the wake-up is considered successful. Otherwise, it is a false wake-up event. The total received power at the origin from the set of active BSs located at $x_{i}$ is

$$
P_{r}=a \sum_{x_{i} \in \Psi} P h_{x_{i}}\left\|x_{i}\right\|^{-\alpha},
$$

which denotes the net interference power if $\Psi$ does not include the serving BS and the interference plus signal power if it includes the serving BS. The probability of having $P_{r} \geq T$ is denoted as the wake-up probability and is defined as

$$
\begin{aligned}
P_{w} & \triangleq \mathbb{P}\left(P_{r} \geq T\right) \\
& =\mathbb{P}\left(P_{r} \geq T \mid b_{0} \in \Psi\right) \mathbb{P}\left(b_{0} \in \Psi\right) \\
& +\mathbb{P}\left(P_{r} \geq T \mid b_{0} \in \Delta\right) \mathbb{P}\left(b_{0} \in \Delta\right) \\
& =P_{s} \cdot \mathbb{P}\left(b_{0} \in \Psi\right)+P_{f} \cdot \mathbb{P}\left(b_{0} \in \Delta\right),
\end{aligned}
$$

where $P_{s}=\mathbb{P}\left(P_{r} \geq T \mid b_{0} \in \Psi\right)$ is the success wake-up probability given that the serving BS is active and $P_{f}=$ $\mathbb{P}\left(P_{r} \geq T \mid b_{0} \in \Delta\right)$ is the probability of having a successful wake-up given that the serving BS is in the set $\Delta$ (idle) which corresponds to the false wake-up probability. Note that $P_{s}$ and $P_{f}$ are derived in the following sections, $\mathbb{P}\left(b_{0} \in \Psi\right)$ and $\mathbb{P}\left(b_{0} \in \Delta\right)$ are the probabilities of having the serving BS in $\Psi$ and $\Delta$, respectively.
The wake-up probability represents the average fraction of devices that, at any time achieve $P_{r} \geq T$. It is also the complementary cumulative distribution function (CCDF) of $P_{r}$. By using (1) we can derive the CCDF of the received power by the device located at the origin, without loss of generality. The wake-up probability can be given by the following theorem:

Theorem 1 (Wake-up probability). In an IoT cellular network, assuming that the BSs constitute a PPP with density $\lambda$ and each BS is transmitting with power $P$, the wake-up probability for a generic device with RF-DC conversion factor a is given by

$$
\begin{aligned}
P_{w}= & \int_{0}^{\infty} \frac{1}{\pi u} \exp [-u T] \\
& \times \exp \left[-\frac{2 \pi^{2} \lambda \delta}{\alpha \tan \left(\frac{2 \pi}{\alpha}\right)}(a P)^{\frac{2}{\alpha}} u^{\frac{2}{\alpha}}\right] \\
& \times \sin \left(\frac{2 \pi^{2} \lambda \delta}{\alpha}(a P)^{\frac{2}{\alpha}} u^{\frac{2}{\alpha}}\right) \mathrm{du} .
\end{aligned}
$$

Proof: See Appendix A

Note that (3) cannot be obtained in closed-form for a general pathloss exponent; however, it can be easily evaluated numerically. Furthermore, a closed-form for (3) exists when $\alpha=4$. In this case, the $\mathrm{CCDF}$ of the amount of received power can be obtained as in Corollary 1 .

Corollary 1. The wake-up probability for the case of $\alpha=4$ can be expressed as:

$$
P_{w}=1-2 \mathrm{Q}\left(\frac{\pi^{2} \sqrt{2} \lambda \delta}{4} \sqrt{\frac{a P}{T}}\right),
$$

where $\mathrm{Q}(z)=\frac{1}{\sqrt{2 \pi}} \int_{z}^{\infty} e^{-\frac{t^{2}}{2}} \mathrm{dt}$ is the Gaussian CCDF.

Note that it can-be seen from (4) that the amount of received power is directly proportional to the spatial density and the transmit power of the BSs, the RF-DC conversion factor of the added wake-up module and the active probability. Therefore, the increased load of interference, which is induced from increasing the density of active BSs, may increase the chance of wake-up.

\section{FAlse Wake-up Probability}

A false wake-up is an event where the device falsely wakesup without any signal transmitted from its serving BS. The false wake-up can be triggered at the device by wake-up signals transmitted by nearby active BSs towards their devices.

\section{A. General Expression}

The probability of false wake-up is the probability that the received power at the desired device exceeds the threshold $T$ given that the serving $\mathrm{BS}$ is not sending a wake-up signal to the device ( $b_{0} \in$ the idle set $\Delta$ ). This probability can be expressed as

$$
P_{f} \triangleq \mathbb{P}\left(P_{r} \geq T \mid b_{0} \in \Delta\right)=\mathbb{P}(I \geq T),
$$


where

$$
I=a \sum_{x_{i} \in \psi \backslash b_{0}} P h_{x_{i}}\left\|x_{i}\right\|^{-\alpha}
$$

is the cumulative power (interference) received from all the other BSs (except the serving BS for the device at the origin, denoted as $b_{0}$ ) which are at a distance $\left\|x_{i}\right\|$ from the device of interest. Since we are excluding the serving BS from the received power field, the unconditional thinning of the PPP of BSs to obtain the set of active BSs is valid.

An important quantity is the distance $r$ separating the device from its serving BS. Since each device communicates with the closest BS, no other BS can be closer than $r$. In other words, all interfering BSs must be farther than $r$. The PDF of the distance between a generic device and the nearest BS is given by [40]:

$$
f_{R}(r)=e^{-\lambda \pi r^{2}} 2 \pi \lambda r .
$$

Conditioning on the nearest BS being at a distance $r$ from the given device, the false wake-up probability averaged over the plan can be derived as

$$
\begin{aligned}
P_{f} & =\int_{r>0} \mathbb{P}(I \geq T \mid r) f_{R}(r) \mathrm{dr} \\
& =\int_{r>0} \mathbb{P}_{r}(I \geq T) e^{-\pi \lambda r^{2}} 2 \pi \lambda r \mathrm{~d} r .
\end{aligned}
$$

The conditioned false wake-up probability, denoted hereafter as $\mathbb{P}_{r}(I \geq T)$, is exactly the CCDF of the interference field $I$ at the device with fixed $r$ when no signal is transmitted from its BS. The CCDF of the conditioned interference field can be obtained by inverse Laplace transform. This calculation follows the same procedure as in the proof of Theorem 1 with modification in the integral bounds. Thus, the Laplace transform of the interference $\mathcal{L}_{I}(s)$ is

$$
\mathcal{L}_{I}(s)=\exp \left[-2 \pi \lambda \delta \int_{r}^{\infty} \frac{v}{1+\frac{1}{s a P} v^{\alpha}} \mathrm{dv}\right] .
$$

After some mathematical manipulations and substituting $u^{\alpha}=$ $\frac{1}{s a P} v^{\alpha}$, we obtain

$\mathcal{L}_{I}(s)=\exp \left\{-\frac{2 \pi \delta \lambda s a P r^{2-\alpha}}{(\alpha-2)}\left[{ }_{2} F_{1}\left(1,1-\frac{2}{\alpha} ; 2-\frac{2}{\alpha} ;-s a P r^{-\alpha}\right)\right]\right\}$.

Note that the function ${ }_{2} F_{1}($.$) is the Gauss hypergeometric$ function and it can be evaluated by numerical methods. In addition, it reduces to simple closed-form expressions for some special values of $\alpha$ [41]. The CCDF of the interference field $I$ can then be obtained from the complex inversion integral formula for Laplace transform using the Euler characterization [42] as

$$
\mathbb{P}_{r}(I \geq T)=\frac{2 e^{c T}}{\pi} \int_{0}^{\infty} \operatorname{Re}\left(\overline{\mathcal{L}}_{I}(c+i u)\right) \cos (u T) \mathrm{du},
$$

where $c$ is a constant and

$$
\overline{\mathcal{L}}_{I}(s)=\frac{1}{s}-\frac{\mathcal{L}_{I}(s)}{s} .
$$

Unfortunately, the manipulation of this equation is not easy and the unavailability of closed form expression for the CCDF of the interference field motivates the search of lower and upper bounds, which will be derived next. The derivation of these bounds follows the approach used in [43] which presents lower and upper bounds on the transmission capacity via lower and upper bounds on SINR outage probability. Our work extends the existing results by deriving bounds for the false wake-up probability via bounds on the interference field itself.

\section{B. False Wake-up Probability with Path Loss Channel Model}

In this section, a baseline model is presented where the only randomness is in the positions of devices and BSs, i.e., there is no fading $\left(h_{x}=1\right)$ and the pathloss is the only parameter affecting the received power at the given device. The impact of fading is addressed in Section $\mathrm{V}-\mathrm{C}$ A lower bound on the false wake-up probability is obtained by partitioning the set of BSs into dominating (near set $\Phi_{T}$ ) and non dominating (far set $\Phi_{T}^{c}$ ) BSs. A BS is dominating if it can individually contribute at least $T$ to $I$ other than the serving BS sending the wake-up signal. The far set is the complement of the near set. The dominating nodes in the near set may be defined geometrically as the BSs located inside a disk centered at the origin of radius $\left(\frac{a P}{T}\right)^{\frac{1}{\alpha}}$ and outside $b(o, r)$ where $b(o, r)$ is the closed ball centered at 0 and of radius $r$ and $r$ is the distance to the nearest BS. For simplicity, we replace the term $\left(\frac{a P}{T}\right)^{\frac{1}{\alpha}}$ with $P_{n}$ in the later derived expressions.

$$
\begin{aligned}
\Phi_{T} & =\left\{x \in \psi \backslash b_{0}, a P\|x\|^{-\alpha} \geq T\right\} \\
& =\left\{x \in \psi \backslash b_{0},\|x\| \leq P_{n}^{\frac{1}{\alpha}}\right\} \\
& =\psi \cap \bar{b}(o, r) \cap b\left(o, P_{n}^{\frac{1}{\alpha}}\right) .
\end{aligned}
$$

Note that if $r$ is higher than $P_{n}^{\frac{1}{\alpha}}$, no BS other than the serving BS will contribute at least $T$ to $I$ and the lower bound is null. We have $I=I_{\Phi_{T}}+I_{\Phi_{T}^{c}}$ where $I_{\Phi_{T}}$ corresponds to the interference from the set $\Phi_{T}$ and $I_{\Phi_{T}^{c}}$ corresponds to the interference from the set $\Phi_{T}^{c}$. The lower bound $\mathbb{P}_{r}(I \geq T)$ can be derived by neglecting the contribution of the far set, which gets tighter as $\alpha$ increases due to the increasing dominance of proximity interferers. Hence we have

$$
\mathbb{P}_{r}(I \geq T)=\mathbb{P}\left(I_{\Phi_{T}}+I_{\Phi_{T}^{c}} \geq T\right) \geq \mathbb{P}\left(I_{\Phi_{T}} \geq T\right)=P_{f_{l}}^{r}
$$

Note that by construction the event $\left\{I_{\Phi_{T}} \geq T\right\}$ is the same as the event $\left\{\Phi_{T} \neq \emptyset\right\}$ which is simply the complement of $\left\{\Phi_{T}=\emptyset\right\}$.

$$
\begin{aligned}
P_{f_{l}}^{r}=1-\mathbb{P}\left(\Phi_{T}=\emptyset\right) & =1-e^{-\lambda\left|\bar{b}(o, r) \cap b\left(o, P_{n}^{\frac{1}{\alpha}}\right)\right|} \\
& =1-e^{-\delta \lambda \pi P_{n}^{\frac{2}{\alpha}}+\delta \lambda \pi r^{2}} .
\end{aligned}
$$

An upper bound for $\mathbb{P}_{r}(I \geq T)$ follows by observing first that the event $\{I<T\}$ requires the near set to be empty and that the contribution of the far set can be replaced by its average using the Markov inequality. To calculate the upper bound, we decompose the conditioned false wake-up event as

$$
\begin{aligned}
\mathbb{P}_{r}(I \geq T) & =\mathbb{P}_{r}\left(\left\{I_{\Phi_{T}} \geq T\right\} \cup\left\{I_{\Phi_{t}^{c}} \geq T\right\}\right. \\
& \left.\cup\left\{I_{\Phi_{T}} \leq T, I_{\Phi_{T}^{c}} \leq T, I_{\Phi_{T}}+I_{\Phi_{T}^{c}} \geq T\right\}\right) .
\end{aligned}
$$


The third event is null and the probability of the remaining events can be written as:

$$
\begin{aligned}
\mathbb{P}_{r}(I \geq T) & =\mathbb{P}_{r}\left(I_{\Phi_{T}} \geq T\right)+\mathbb{P}_{r}\left(I_{\Phi_{T}^{c}} \geq T\right) \\
& -\mathbb{P}_{r}\left(I_{\Phi_{T}} \geq T\right) \mathbb{P}_{r}\left(I_{\Phi_{T}^{c}} \geq T\right) \\
& =P_{f_{l}}^{r}+\left(1-P_{f_{l}}^{r}\right) \mathbb{P}_{r}\left(I_{\Phi_{T}^{c}} \geq T\right) .
\end{aligned}
$$

To upper bound $\mathbb{P}_{r}\left(I_{\Phi_{T}^{c}} \geq T\right)$ we use the Markov inequality as follows:

$$
\begin{aligned}
\mathbb{P}_{r}\left(I_{\Phi_{T}^{c}} \geq T\right) & \stackrel{(a)}{\leq} \frac{1}{T} \mathbb{E}\left[I_{\Phi_{T}^{c}}\right] \\
& =\frac{1}{T} \mathbb{E}\left[\sum_{x_{i} \in \psi \cap \bar{b}\left(o, P_{n}^{\frac{1}{\alpha}}\right)} a P\left\|x_{i}\right\|^{-\alpha}\right] \\
& \stackrel{(b)}{=} \frac{1}{T} \int_{\left(P_{n}\right)^{\frac{1}{\alpha}}}^{\infty} a P v^{-\alpha} 2 \pi \lambda v \mathrm{dv}=\frac{2 \pi \lambda}{\alpha-2} P_{n}^{\frac{2}{\alpha}},
\end{aligned}
$$

where (a) follows from the Markov inequality and (b) from the Campbell's theorem [39]. The conditioned upper bound of the false wake-up probability can be expressed as

$$
P_{f_{u}}^{r}=\left(1-e^{-\delta \lambda \pi P_{n}^{\frac{2}{\alpha}}+\delta \lambda \pi r^{2}}\right)+\frac{2 \pi \lambda \delta}{\alpha-2} P_{n}^{\frac{2}{\alpha}} e^{-\delta \lambda \pi P_{n}^{\frac{2}{\alpha}}+\delta \lambda \pi r^{2}} .
$$

The unconditioned lower and upper bounds $P_{f_{l}}$ and $P_{f_{u}}$ for the CCDF of the interference field can be derived by averaging over $r$ the conditioned bounds. Note that the integral is between 0 and $P_{n}^{\frac{1}{\alpha}}$ because $r$ is always less than $P_{n}^{\frac{1}{\alpha}}$. Thus, the false wake-up probability can be lower and upper bounded respectively, by

$$
\begin{gathered}
P_{f_{l}}=1-e^{-\lambda \pi P_{n}^{\frac{2}{\alpha}}}+\frac{1}{1-\delta}\left[e^{-\lambda \pi P_{n}^{\frac{2}{\alpha}}}-e^{-\delta \lambda \pi P_{n}^{\frac{2}{\alpha}}}\right] \\
P_{f_{u}}=1-e^{-\lambda \pi P_{n}^{\frac{2}{\alpha}}}+\frac{1}{1-\delta}\left(1-\frac{2 \pi \delta \lambda}{\alpha-2} P_{n}^{\frac{2}{\alpha}}\right)\left[e^{-\lambda \pi P_{n}^{\frac{2}{\alpha}}}-e^{-\delta \lambda \pi P_{n}^{\frac{2}{\alpha}}}\right]
\end{gathered}
$$

\section{False Wake-up Probability with Fading Channel Model}

We now extend the analysis to include the fading $h$ which follows an exponential distribution with unit mean. The same concept as in Section $\mathrm{V}-\mathrm{B}$ is used and the near and far sets $\Phi_{T}$ and $\Phi_{T}^{c}$ are defined as follows:

$$
\begin{array}{ll}
\Phi_{T}=\left\{x \in \psi \backslash b_{0}, a P h\|x\|^{-\alpha} \geq T\right\} & \text { (near set). } \\
\Phi_{T}^{c}=\left\{x \in \psi \backslash b_{0}, a P h\|x\|^{-\alpha}<T\right\} & \text { (far set). }
\end{array}
$$

The conditioned lower bound $P_{f_{l}}^{r}$ can be calculated by evaluating the probability that the near set $\Phi_{T}$ is empty using the Laplace functional. After some mathematical manipulations we obtain the conditioned lower bound as in the following lemma.

Lemma 1. In an IoT cellular network with a pathloss exponent $\alpha$ and where the density of BSs is $\lambda$, the conditioned false wake-up probability of a generic device can be lower bounded by

$P_{f_{l}}^{r}=1-\exp \left\{-\pi \lambda \delta\left[-r^{2} e^{-\frac{r^{\alpha}}{P_{n}}}+P_{n}^{\frac{2}{\alpha}} \Gamma\left(1+\frac{2}{\alpha}, \frac{r^{\alpha}}{P_{n}}\right)\right]\right\}$, where $\Gamma[s, x]=\int_{x}^{\infty} t^{s-1} e^{-t} \mathrm{dt}$ denotes the incomplete gamma function.

Proof: See Appendix B.

The upper bound is derived as follows:

$$
\begin{aligned}
\mathbb{P}_{r}(I \geq T) & =\mathbb{P}\left(I_{\Phi_{T}} \geq T\right)+\mathbb{P}\left(I \geq T \mid I_{\Phi_{T}}<T\right) \mathbb{P}\left(I_{\Phi_{T}}<T\right) \\
& =P_{f_{l}}^{r}+\mathbb{P}\left(I \geq T \mid I_{\Phi_{T}} \leq T\right)\left(1-P_{f_{l}}^{r}\right)=P_{f_{u}}^{r} .
\end{aligned}
$$

After some mathematical derivations, the conditioned upper bound for the CCDF denoted as $P_{f_{u}}^{r}$ can be expressed as in Lemma 2.

Lemma 2. The conditioned false wake-up probability can be upper bounded by:

$$
\begin{aligned}
P_{f_{u}}^{r} & =P_{f_{l}}^{r}+\frac{2 \pi \lambda \delta}{\alpha-2}\left[P_{n} r^{2-\alpha}\left(1-e^{-\frac{r^{\alpha}}{P_{n}}}\left(1+\frac{r^{\alpha}}{P_{n}}\right)\right)\right. \\
& \left.+P_{n}^{\frac{2}{\alpha}} \Gamma\left(1+\frac{2}{\alpha}, \frac{r^{\alpha}}{P_{n}}\right)\right]\left(1-P_{f_{l}}^{r}\right) .
\end{aligned}
$$

\section{Proof: See Appendix C}

The unconditioned lower and upper bounds for the CCDF $P_{f_{l}}$ and $P_{f_{u}}$ of the interference field can be finally derived by averaging over $r$ the conditioned bounds in 22) and (24). Thus, the false wake-up probability can be bounded by 25 and 26 .

$$
\begin{aligned}
P_{f_{l}} & =1-\pi \lambda \int_{0}^{\infty} \exp \left\{-\pi \lambda \delta\left[z\left(\frac{1}{\delta}-e^{-\frac{z^{\frac{\alpha}{2}}}{P_{n}}}\right)\right.\right. \\
& \left.\left.+P_{n}^{\frac{2}{\alpha}} \Gamma\left(1+\frac{2}{\alpha}, \frac{z^{\frac{\alpha}{2}}}{P_{n}}\right)\right]\right\} \mathrm{d} \mathrm{z} .
\end{aligned}
$$

$$
\begin{aligned}
P_{f_{u}} & =P_{f_{l}}+\pi \lambda \int_{0}^{\infty} \frac{2 \pi \lambda \delta}{\alpha-2}\left[P_{n} z^{1-\frac{\alpha}{2}}\left(1-e^{-\frac{z^{\frac{\alpha}{2}}}{P_{n}}}\left(1+\frac{z^{\frac{\alpha}{2}}}{P_{n}}\right)\right)\right. \\
& \left.+P_{n}^{\frac{2}{\alpha}} \Gamma\left(1+\frac{2}{\alpha}, \frac{z^{\frac{\alpha}{2}}}{P_{n}}\right)\right] \exp \left\{-\pi \lambda \delta\left[z\left(\frac{1}{\delta}-e^{-\frac{z^{\frac{\alpha}{2}}}{P_{n}}}\right)\right.\right. \\
& \left.\left.+P_{n}^{\frac{2}{\alpha}} \Gamma\left(1+\frac{2}{\alpha}, \frac{z^{\frac{\alpha}{2}}}{P_{n}}\right)\right]\right\} \mathrm{d} z .
\end{aligned}
$$

\section{SUCCESS WAKE-UP PROBABILITy}

In order to successfully wake-up, the device should receive a power $P_{r}$ greater than a threshold $T$ when the serving BS is transmitting the wake-up signal. If $P_{r}$ is below $T$, it remains in the sleep mode. A simple thinning of the BSs point process to get the set of active BSs is not valid in this case since it may also turn off the serving BS. To address this problem, we introduce a conditional thinning [44] of the received power field predicted on a wake-up signal transmitted from the serving BS to a typical active device. We assume that a typical device connects to the nearest BS. Conditioned on this connection, each BS in the received power field transmits independently with a probability $\delta$ and is idle with a probability $1-\delta$. Since partitioning is done independently, both the set of active BSs $\Psi$ and the set of idle BSs $\Delta$ may 
contain the serving BS. This will make it harder to analyze the system model since we do not have a priori knowledge about the serving BS and hence it is impossible to isolate the received power field.

The desired success wake-up probability $\mathbb{P}\left(P_{r} \geq T \mid b_{0} \in \Psi\right)$ can be calculated by evaluating the unconditioned success probability which corresponds to the probability that the received power exceeds a predefined threshold without conditioning on having the serving $\mathrm{BS} b_{0}$ active. This is exactly the wake-up probability $P_{w}$ derived is (2) and (3).

The distances from the device of interest to the nearest BS in the set $\Psi$ and the nearest BS in $\Delta$ are denoted as $R_{1}$ and $R_{2}$, respectively. Therefore, the distance from the device of interest to its serving $\mathrm{BS}$ is

$$
R_{0}=\min \left\{R_{1}, R_{2}\right\}
$$

The probability of having the serving BS in the set $\Psi$ can be calculated as

$$
\begin{aligned}
\mathbb{P}\left(b_{0} \in \Psi\right) & =\mathbb{P}\left(R_{0}=R_{1}\right)=\mathbb{P}\left(R_{1}<R_{2}\right) \\
& =\mathbb{E}_{R_{1}}\left[\mathbb{P}\left(R_{2}>R_{1} \mid R_{1}\right)\right] \\
& =\int_{0}^{\infty} e^{-\lambda \pi(1-\delta) R_{1}^{2}} 2 \pi \lambda \delta R_{1} e^{-\lambda \pi \delta R_{1}^{2}} \mathrm{dR}_{1}=\delta .
\end{aligned}
$$

Therefore $\mathbb{P}\left(b_{0} \in \Delta\right)=1-\delta$.

The success wake-up probability $P_{s}$ can be calculated as

$$
P_{s}=\frac{1}{\delta} P_{w}-\frac{1-\delta}{\delta} P_{f}
$$

where $P_{w}$ is derived in Section IV] and lower and upper bounds are found for $P_{f}$ in Section $\mathrm{V}$. Therefore, lower and upper bounds can be derived for $P_{s}$ for both pathloss and fading models.

\section{SINR UPLINK COVERAGE PROBABILITy}

We assume that after wake-up, each device will initiate uplink communication with its serving BS. We define the SINR uplink coverage probability $P_{c}$ as the probability that the received SINR at the serving BS is higher than a predefined threshold. Therefore, a device can establish a successful communication link with its serving BS under two conditions:

1) The amount of received power by the device in the downlink given that its serving BS is transmitting a wake-up signal is greater than the required amount of power to switch the device from the sleep mode to the active mode. The probability that this condition holds true (i.e., success wake-up probability $P_{s}$ ) has been derived in Section VI

2) After successful wake-up, the level of the received SINR from uplink transmission at the serving BS is greater than a predefined threshold in order to guarantee an acceptable quality of service. The probability of this event is referred to as the SINR uplink coverage probability $P_{c}$ and is derived in this section.

The overall success probability $\eta$ is defined as the probability that both of the aforementioned conditions are satisfied. By virtue of full channel inversion power control, these two events become independent. Thus, the overall success probability is

$$
\eta=P_{s} \cdot P_{c}
$$

The failure probability $\theta$ is the probability that the device is not able to wake-up or has not achieved uplink SINR $\geq \tau$. Therefore, the probability of failure is

$$
\theta=\left(1-P_{s}\right)+P_{s} \cdot\left(1-P_{c}\right) .
$$

In this section, we derive the distribution of the transmission power for a typical device, then we derive the SINR uplink coverage probability.

\section{A. Transmit Power Analysis}

Based on the system model, the devices are assumed to perform full channel inversion in order to satisfy a power level requirement $\rho$ at the serving BS. We assume also that the BSs are dense enough such that each of the devices can invert its path loss towards the closest BS almost surely, so the maximum transmit power of the devices is not a binding constraint for transmission. We define the required amount of transmit power of a device as

$$
\gamma=\rho \cdot R^{\alpha}
$$

where $R$ is the distance to the serving BS from the device. The distribution of the transmit power of a generic active device is obtained using the following lemma.

Lemma 3. In a Poisson cellular network with full channel inversion power control and with BS power level requirement $\rho$, the PDF of the transmit power of a device in the uplink is given by

$$
f_{\gamma}(t)=\frac{2 \pi \lambda}{\alpha \rho}\left(\frac{t}{\rho}\right)^{\frac{2}{\alpha}-1} \exp \left[-\pi \lambda\left(\frac{t}{\rho}\right)^{\frac{2}{\alpha}}\right] .
$$

The moments of the transmit power can be obtained as

$$
\mathbb{E}\left[\gamma^{c}\right]=\frac{\rho^{c} \Gamma\left(\frac{c \alpha}{2}+1\right)}{(\pi \lambda)^{\frac{c \alpha}{2}}},
$$

where $c \in \mathbb{R}^{+}$and $\Gamma(a)=\int_{0}^{\infty} t^{a-1} e^{-t} \mathrm{dt}$ is the gamma function.

Proof: See [45].

Lemma 3 shows that the smaller the power level requirement $\rho$, the lower is the transmit power of the devices. This is because as $\rho$ increases, the devices are required to transmit at a higher power. Lemma 3 also shows that the average transmit power decreases with $\lambda$. That is, as the spatial density of BSs increases, the distance between a device and the corresponding serving BS decreases and hence, a lower transmit power is required.

\section{B. SINR Analysis}

The SINR uplink coverage probability $P_{c}$ is defined as the probability that the received SINR at a BS is higher than a predefined threshold $\tau$. It can be mathematically defined as

$$
P_{c}=\mathbb{P}[\mathrm{SINR} \geq \tau] .
$$


Without loss of generality, the SINR used in (35) can be defined for a BS located at the origin as

$$
\mathrm{SINR}=\frac{\rho h}{\sum_{y_{i} \in \tilde{\psi}} \gamma_{i} h_{y_{i}}\left\|y_{i}\right\|^{-\alpha}+\sigma^{2}}=\frac{\rho h}{I+\sigma^{2}} .
$$

where the useful signal power is equal to $\rho h$ due to the full channel inversion power control, $h$ and $h_{y_{i}}$ are the smallscale fading coefficients between the BS and its served device $y_{0}$ and its interfering devices, respectively. $\tilde{\psi}$ is the point process, representing the interfering devices and $I$ denotes the aggregate interference at the considered BS from the set of interfering devices transmitting at the same time and on the same resources. Using the SINR expression given in (36) and the definition in 35 , the SINR uplink coverage probability can be calculated as

$$
\begin{aligned}
P_{c}=\mathbb{P}[\operatorname{SINR} \geq \tau] & =\mathbb{E}_{I}\left[\mathbb{P}\left[h \geq \frac{\tau I+\tau \sigma^{2}}{\rho}\right]\right] \\
& =e^{-\frac{\tau \sigma^{2}}{\rho}} \mathcal{L}_{I}\left(\frac{\tau}{\rho}\right) .
\end{aligned}
$$

where $\mathcal{L}_{I}(\cdot)$ denotes the Laplace transform (LT) of the aggregate interference $I=\sum_{y_{i} \in \tilde{\psi}} \gamma_{i} h_{y_{i}}\left\|y_{i}\right\|^{-\alpha}$.

The point process $\tilde{\psi}$ of the interfering devices represents the set of active devices at a certain time slot, where "active devices" refers to devices that have received sufficient power to wake-up and are ready for transmission. We consider that at any time instant each BS has a single device served on a given channel resource. That is, in each cell, the active devices are scheduled according to a specific scheduling scheme and only one device initiates uplink communication at a time. Note that in the uplink, the interfering devices do not constitute a PPP due to the correlations among them. These correlations are induced by the unique channel assignment per device in each BS. However, for analytical tractability, we approximate the active interfering devices set to a PPP with spatial density equal to the density of the BS set multiplied by the active probability which corresponds to the wake-up probability $P_{w}$ in a given time slot. We will consider also that the transmit powers of the interfering devices are iid. These approximations have been used and validated in the literature in [45]-[47]. Note that the active probability $P_{w}$ calculated in (3) represents the fraction of devices that have successfully woken-up and does not differentiate between a success and a false wake-up. Even though the correlations between the interfering devices are ignored in the PPP assumption of the interferes set $\tilde{\psi}$, the unique channel assignment brings further correlation between the locations of the interferes and the considered BS at the origin. Therefore, the average interference from any single device is strictly less than $\rho$ and the aggregate interference received at the tagged $\mathrm{BS}$ can be written as

$$
I=\sum_{y_{i} \in \tilde{\psi} \backslash y_{0}} \mathbf{1}\left(\gamma_{i}\left\|y_{i}\right\|^{-\alpha}<\rho\right) \gamma_{i} h_{y_{i}}\left\|y_{i}\right\|^{-\alpha} .
$$

The indicator function captures the correlation between the interfering devices and the considered BS and ensures that the average interference received at a BS is strictly less than $\rho$. The SINR uplink coverage probability can be finally given by the following theorem.
Theorem 2. In a IoT cellular network with channel inversion power control in the uplink, assuming that the interfering devices constitute a PPP, the SINR coverage probability is given by

$$
P_{c}=\exp \left\{-\frac{2 P_{w} \tau}{(\alpha-2)}\left[{ }_{2} F_{1}\left(1,1-\frac{2}{\alpha} ; 2-\frac{2}{\alpha} ;-\tau\right)\right]-\frac{\tau \sigma^{2}}{\rho}\right\} .
$$

where ${ }_{2} F_{1}($.$) is the Gauss hypergeometric function.$

Proof: See Appendix D

We can see that the SINR uplink coverage probability highly depends on $P_{w}$ or the portion of devices that have wokenup. In this case, after fixing a device per BS, a portion of these devices is transmitting and increasing the interference level in the network. The portion of these interferers is not however affecting the distance to their serving BSs and their transmit powers. Therefore, the SINR uplink coverage probability decreases rapidly with the increase of the wake-up probability $P_{w}$. As a special case, assuming that $\alpha$ is 4 , the coverage probability reduces to

$$
P_{c}=\exp \left[-P_{w} \sqrt{\tau} \arctan (\tau)-\frac{\tau \sigma^{2}}{\rho}\right] .
$$

Equation ( 40 ) shows that the decrease of the SINR uplink coverage probability is exponentially fast. By plugging (4) in (40), the coverage probability becomes

$$
P_{c}=\exp \left[-\left(1-2 \mathrm{Q}\left(\frac{\pi^{2} \sqrt{2} \lambda}{4} \sqrt{P_{n}}\right)\right) \sqrt{\tau} \arctan (\tau)-\frac{\tau \sigma^{2}}{\rho}\right] .
$$

\section{ENERGY EFFICIENCY ANALYSIS}

In this section, we aim to evaluate the energy efficiency gains achieved by the introduction of the proposed RF wakeup solution. Since the energy used in the UL transmission is independent of the energy collected from the RF wake-up signal and used to wake-up the device, it will not affect the wake-up process and thus it is not considered in the energy efficiency analysis. In order to quantify the consumed energy of an IoT device with RF wake-up, we consider a time-slotted system with slot duration $\Delta t$. The time slot is partitioned into two sub-slots: active sub-slot (with duration $T_{\mathrm{ON}}$ ) and sleep sub-slot (with duration $T_{\text {sleep }}$ ). The percentage of time in which the device is active is denoted as $D_{\mathrm{ON}}$. The added wake-up receiver will add extra power consumption to the device in both the active and sleep modes and, thus, the overall power consumption is the sum of the main circuit's power along with the power dissipated in the wake-up receiver. The power consumed in the active and sleep modes of an RF wakeup device are denoted, respectively, as $P_{\mathrm{RF} F_{-} \mathrm{ON}}$ and $P_{\mathrm{RF} F_{-} \text {sleep }}$ :

$$
\begin{array}{r}
P_{\mathrm{RF} \text { _ON }}=P_{\text {main_ON }}+P_{\text {Wur_ON }} \\
P_{\mathrm{RF} \text { _sleep }}=P_{\text {main_sleep }}+P_{\text {Wur_sleep }},
\end{array}
$$

where $P_{\text {main_ON }}$ and $P_{\text {main_sleep }}$ are the active and sleep mode power consumptions of the main circuit and $P_{\text {Wur_ON }}$ and $P_{\text {Wur_sleep }}$ are those of the added wake-up receiver.

In order to have a successful wake-up and to transit to the active mode, an RF wake-up device requires the reception of a wake-up signal during the active sub slot $T_{\mathrm{ON}}$. However, 
TABLE II

SiMULATION PARAMETERS

\begin{tabular}{|l|l|}
\hline Parameter & Value \\
\hline BS's transmit power $P$ & $46 \mathrm{dBm}$ \\
\hline BS's density $\lambda\left(\times 10\left(0.5^{2} \pi\right)^{-1}\right)$ & $1,2,5 \mathrm{BS} / \mathrm{km}^{2}$ \\
\hline Path loss exponent $\alpha$ & $3.7,4,4.4$ \\
\hline RF-DC conversion factor $a$ & $0.2,0.5,1$ \\
\hline Active probability $\delta$ & $0.2,0.5,1$ \\
\hline
\end{tabular}

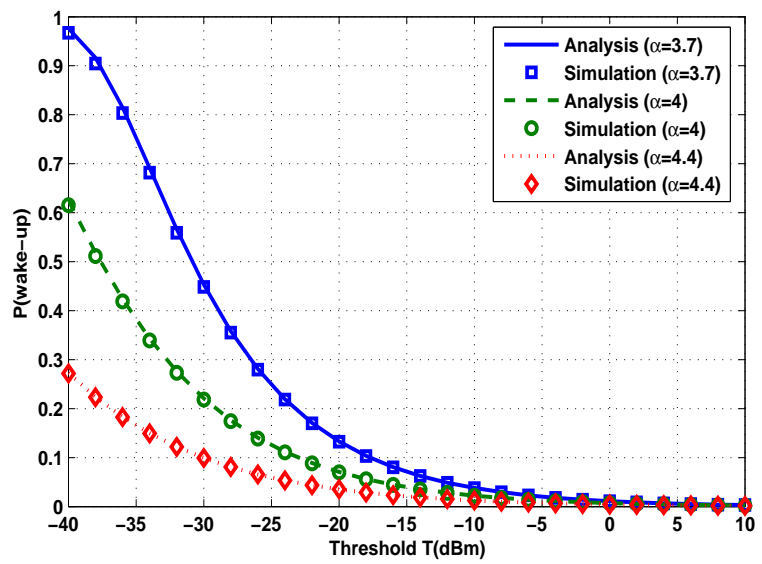

Fig. 5. Wake-up probability as function of $T$ for different values of $\alpha$.

because of the statistical channel effects, the RF wake-up device may not always receive enough power during $T_{\mathrm{ON}}$. As presented in Section VI an RF wake-up device has a probability $P_{s}$ to wake-up successfully in the active sub-slot $T_{\mathrm{ON}}$. In addition, the device may experience a false wake-up with probability $P_{f}$ when sufficient power is received at the device in the sleep sub-slot $T_{\text {sleep }}$. Therefore, the total energy consumption of an RF wake-up device during the slot duration $\Delta t$ can be estimated as follows:

$$
\begin{aligned}
E_{\mathrm{RF}} & =\Delta t \cdot\left[\left(P_{s} \cdot D_{\mathrm{ON}}+P_{f} \cdot\left(1-D_{\mathrm{ON}}\right)\right) P_{\mathrm{RF}_{-} \mathrm{ON}}\right. \\
& +\left(\left(1-P_{s}\right) \cdot D_{\mathrm{ON}}+\left(1-P_{f}\right) \cdot\left(1-D_{\mathrm{ON}_{1}}\right) \cdot P_{\mathrm{RF}_{-} \text {sleep }}\right],
\end{aligned}
$$

where $P_{\mathrm{RF}_{-} \mathrm{ON}}$ and $P_{\mathrm{RF}_{-} \text {sleep }}$ are given in 42 and 43 and bounds for $P_{s}$ and $P_{f}$ are given in 29) and 25, 26, respectively.

To demonstrate the superiority of the proposed RF wake-up solution, we compare it with the standard "scheduled wakeup" mechanism that is based on the duty cycling technique. A device employing the scheduled wake-up technique is internally programmed to wake-up at each active sub-slot $T_{\mathrm{ON}}$ and does not require the integration of an extra wake-up receiver. Thus, the power consumed in the active mode $P_{\text {sch_ON }}$ and sleep mode $P_{\text {sch_sleep }}$ are exactly the power of the main circuit $P_{\text {main_ON }}$ and $P_{\text {main_sleep }}$ respectively; therefore, the overall energy consumption of a scheduled wake-up device can be expressed as:

$$
E_{\mathrm{sch}}=\Delta t \cdot\left[D_{\mathrm{ON}} \cdot P_{\mathrm{sch} \_\mathrm{ON}}+\left(1-D_{\mathrm{ON}}\right) \cdot P_{\text {sch_sleep }}\right] .
$$

\section{NumericAl Results AND Discussions}

In this section, we present performance results obtained from the proposed framework. We focus on the trade-offs

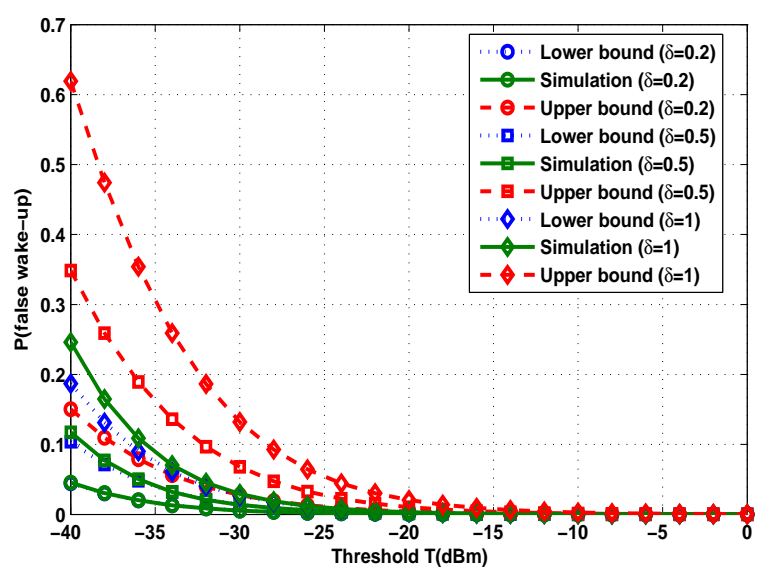

Fig. 6. False wake-up probability with fading as function of $T$ for different values of $\delta$.

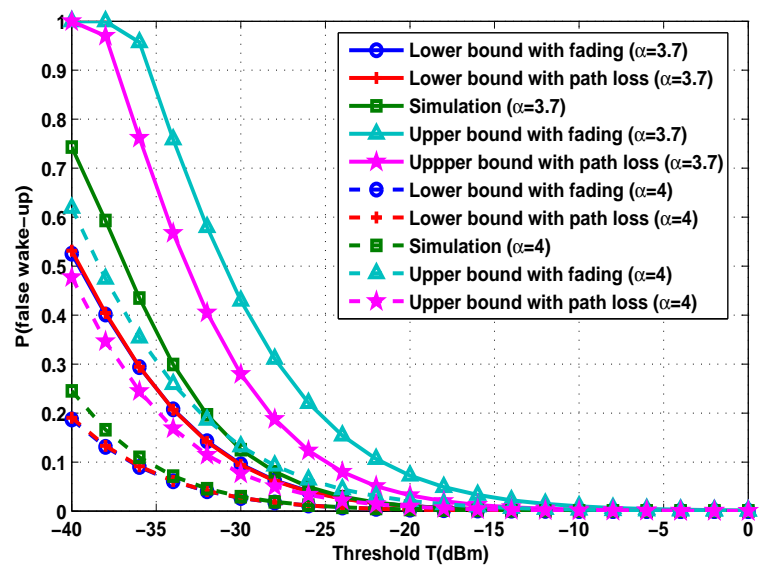

Fig. 7. False wake-up probability for the pathloss and the fading models as function of $T$ for different values of $\alpha$.

between the success and false wake-up probabilities. In addition, we show how the different network parameters affect the performance metrics and the energy efficiency. The scenario under consideration is composed of a set of BSs and a set of devices distributed following two independent PPPs over the network. Unless otherwise stated, the transmit power of BSs is assumed to be $P=46 \mathrm{dBm}$ while the thermal noise is set to 0 (interference-limited scenario). An independent and identically distributed Rayleigh fading with unit mean for its power is considered for all links except the baseline scenario presented in Section V-B The simulation parameters are summarized in Table II It is worth noting that the effect of the RF-DC conversion factor is equivalent to increasing the threshold $T$ at the device by a factor of $a^{-1}$. That is varying $a$ only scales the resulting figures when plotted against $T$.

Fig. 5 illustrates the effect of increasing the pathloss exponent $\alpha$ on the wake-up probability. This figure validates our analytical model for the wake-up probability as it can be seen that the gap between the analytical results and those obtained from Monte-Carlo simulations is tight for different values of $\alpha$. Fig. 6 presents the analytical bounds and the simulation results for the false wake-up probability with fading for different BS 


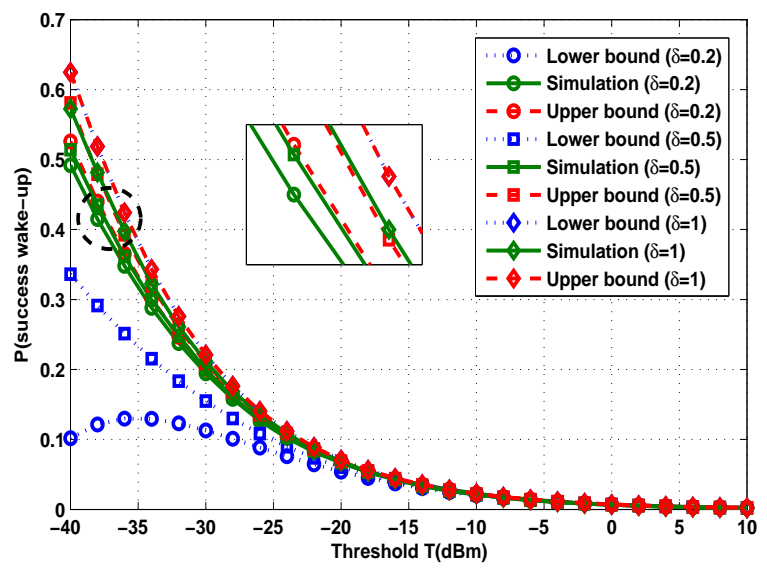

Fig. 8. Success wake-up probability as function of $T$ for different values of $\delta$.

active probabilities $\delta$. From this figure, we observe that the lower bound is closer to the actual performance (simulations) than the upper bound. In the conducted derivations, the upper bound is loose because of the Markov inequality used to bound the term $\mathbb{P}\left(I \geq T \mid I_{\Phi_{T}} \leq T\right)$. A better bound may be obtained by using the Chebyshev's inequality or the Chernoff bound but at the cost of more complexity. The upper bound diverges as $\alpha \downarrow 2$ since the average interference contribution from the far set diverges.

In Fig. 7, we compare the analytical bounds of the false wake-up probability with fading and the false wake-up probability in the baseline scenario where only the pathloss is considered for different values of the pathloss exponent $\alpha$. From this figure, we observe that the lower bounds are almost the same for the pathloss scenario and the scenario with fading. However, the upper bounds differ and the pathloss scenario insures a tighter upper bound by omitting the randomness of the fading. Therefore, by neglecting the fading effect, we can achieve similar results for the lower bound and better results for the upper bound with much more simplified expressions and less complicated analysis. Fig. 8 presents the analytical bounds and the simulation results for the success wake-up probability for different BS active probabilities $\delta$. From this figure, we observe that the lower bound is loose since it follows from the upper bound of the false wake-up probability which is based on the Markov inequality. The upper bound, however, is much closer to the actual distribution obtained from the simulations. By comparing Fig. 8 and Fig. 6 we can note that $\delta$ has higher impact on the false wake-up probability than the success wake-up probability. This makes sense since $\delta$ represents the portion of active neighboring BSs which cause the false wake-up while the success wake-up is mainly caused from the wake-up signal of the serving BS. Therefore, tuning $\delta$ may be an effective solution to reduce the false wakeup probability while ensuring an acceptable success wake-up probability level.

Fig. 9 illustrates the effect of increasing the pathloss exponent $\alpha$ on the SINR uplink coverage probability $P_{c}$. This figure validates our analytical model for the SINR uplink coverage probability as it can be seen that the gap between

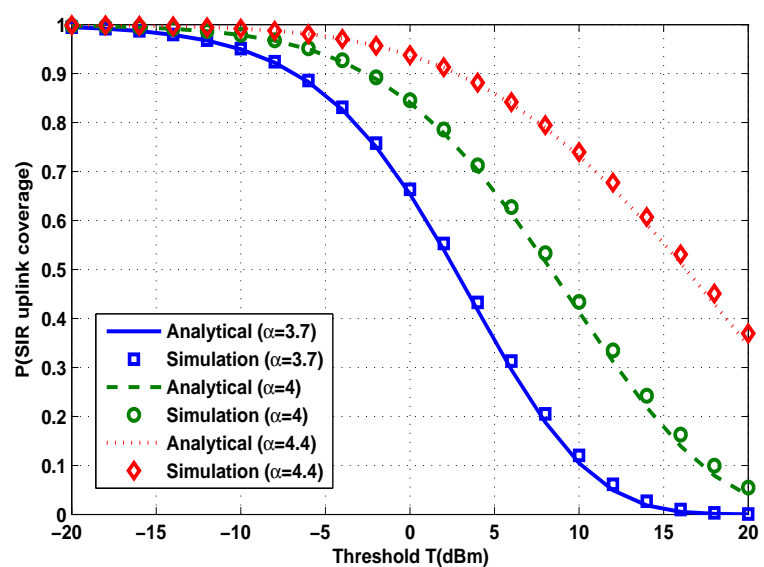

Fig. 9. SINR coverage probability as function of $\tau$ for different values of $\alpha$.

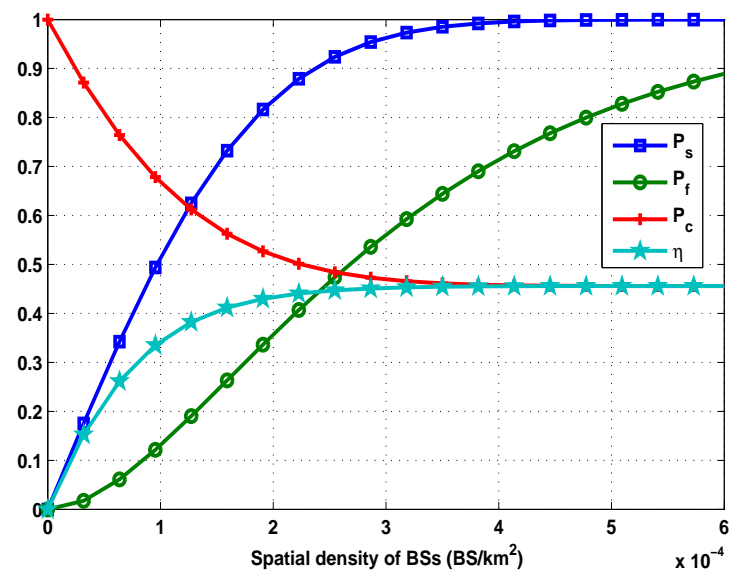

Fig. 10. Success wake-up, false wake-up, SINR uplink coverage probabilities and overall success probability as function of $\lambda$.

the analytical results and those obtained from Monte-Carlo simulations is tight for different values of $\alpha$. From this figure we can note that the SINR coverage probability increases when the pathloss exponent increases. This result can be justified by the following: In an uplink scenario with full channel inversion power control, the useful signal $(\rho h)$ is not affected with the variation of the pathloss which is inverted in the transmit power of the device towards its serving BS. However, the power of the interfering signals is affected when $\alpha$ varies, and for higher $\alpha$ the interfering signals powers are decreasing. Therefore, the overall SINR coverage probability is improved. In addition, the wake-up probability $P_{w}$ decreases when the pathloss exponent increases (see Fig. 5). Therefore, the density of the interfering devices is less and the SINR uplink coverage probability is further improved.

The effect of varying the BS active probability is similar to that of varying the spatial density of BSs which is illustrated on both the success wake-up and the false wake-up probabilities as well as the SINR uplink coverage probability and the overall success probability in Fig. 10. It is worth noting that the effect of increasing the spatial density has the following consequences: 
1) It reduces the distance between the device and its serving $\mathrm{BS}$ and increases the number of available sources which in turn increases the amount of received power per device.

2) On the other hand, the device will acquire higher chance to falsely wake-up due to the increase in the density of other BSs.

3) In addition, more devices will wake-up and the device will experience more interference in the uplink due to the increase of the density of devices that will transmit after wake-up (i.e., density of the point process $\tilde{\psi}$ which is equal to $\left.P_{w} \lambda\right)$. In addition, these interferers become closer. This increase in the interference degrades the SINR at the receiving BS since the useful signal is always constant (i.e., $\rho$ ).

Consequently, as can be seen in Fig. 10, while the SINR uplink coverage probability $P_{c}$ falls with increasing $\lambda$, the success and the false wake-up probabilities (implicitly the wake-up probability $P_{w}$ ) increase. However, it can also be seen that the increase of the success wake-up probability and the false wake-up probability dominates the deterioration of $P_{c}$ and thus the overall success $\eta$ probability increases. This happens up to some point after which deploying more BSs does not have significant effect on the overall performance where $P_{c}, P_{s}$ and $P_{f}$ become almost constant. This behavior can be explained as follows: When the spatial density becomes very high, the expected amount of transmit power becomes very low whereas the power available for wake-up becomes very high. Therefore, the success wake-up probability and the false wake-up probability approach 1 . Furthermore, when the wake-up probability approaches 1 , the SINR uplink coverage probability $P_{c}$ becomes independent of $\lambda$ and approaches a constant level.

From the obtained results, it can be seen that the success and the false probabilities are correlated. Hence, it is important to find a compromise between them to maintain an acceptable performance level. The main challenge is to achieve simultaneously high success probability and low false probability levels. A possible solution is to efficiently tune the network parameters to achieve the required performance while optimizing the gap between these probabilities. Unfortunately, the tuning of these parameters is not sufficient to reduce the high false probability because of the correlation between the success and the false probabilities; therefore, further investigation is required. ID-based wake-up schemes would be a suitable candidate solution to reduce the high false probability. For these solutions, the wake-up signal contains the desired destination address. Therefore, only the device with matching address will wake-up; yet, this comes with additional hardware complexity and a limited increase in energy consumption.

Fig. 11 presents the energy consumption results of the proposed RF wake-up solution compared to "scheduled wakeup" based on the analysis in Section VIII Note that the considered success and false wake-up probabilities in Fig. 11 are taken from the results presented above for specific combinations of the system parameters. We consider that the active sub-slot $T_{\mathrm{ON}}$ is $1 \mathrm{~min}$ for both scheduled and RF wakeup options. We consider also that the sleep sub-slot of the

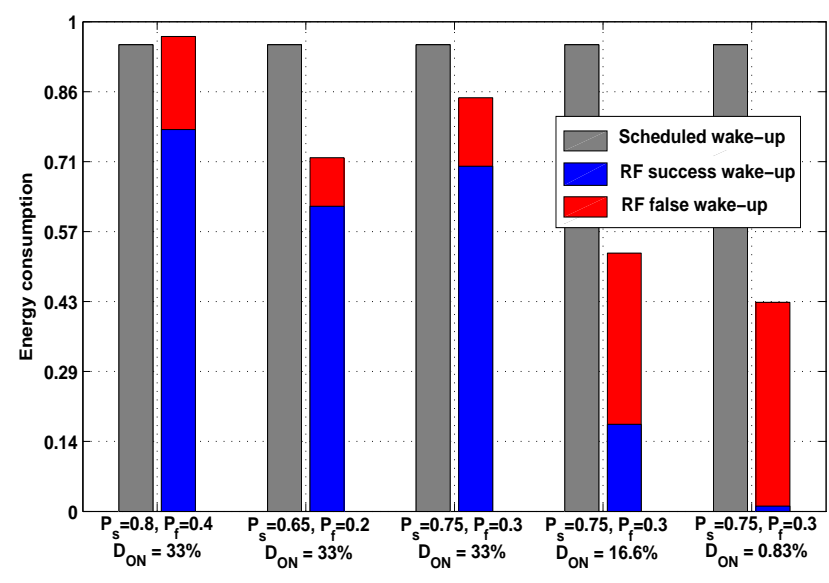

Fig. 11. Energy consumption of a scheduled wake-up device and an RF wakeup device for different values of the active cycle percentage $D_{\mathrm{ON}}$ and the success and false wake-up probabilities.

scheduled wake-up is equal to $2 \mathrm{~min}$. On the other hand, the RF wake-up devices have several values of the sleep subslot corresponding to different IoT use cases with $T_{\text {sleep }}$ of $\{2,5,120\} \min$, i.e., the RF wake-up device has an active time percentage of $33.3 \%, 16.6 \%$ and $0.83 \%$, respectively.

Energy consumption results presented in Fig. 11 show that RF wake-up can achieve significant gains over scheduled wake-up for given design parameters. The gain is highest for IoT use cases that require lower active time percentages. This translates in estimated lifetimes that are longer than those achievable in scheduled-based devices. However, this comes at the cost of less reliability since an RF wake-up device has a probability $P_{s}<1$ to have a successful wake-up. In addition, because the proposed wake-up solution is "broadcast-based", a device can falsely wake up and consume extra energy even if no wake-up signal is intended for it; yet, even with this overhead, notable gains can still be achieved for low values of $D_{\mathrm{ON}}$ as shown in Fig. 11

\section{CONClusion}

The proposed work considers a novel approach to reduce the power consumption and improve the energy efficiency of IoT devices over cellular networks. Instead of wakingup from sleep state on a timer basis and wasting power if the device has no data to transmit, the IoT devices wake-up after receiving dedicated RF signals from their serving BSs. This solution is well suited to the infrequent transmissions in various IoT use cases where a periodic wake-up is not needed and thus highly power inefficient. We have used stochastic geometry to model, analyze and evaluate the performance of the network when this RF wake-up solution is introduced. We have obtained expressions for the wake-up probability, in addition to lower and upper bounds for the false and success wake-up probabilities and a closed-form expression of the SINR uplink coverage probability. The numerical results based on derived expressions show that the proposed wakeup scheme can be a viable solution to enhance the energy 
efficiency of IoT over cellular. However, this comes at the cost of increased false wake-up rate.

\section{APPENDIX}

\section{A. Proof of Theorem 1}

To derive the wake-up probability $P_{w}$ as the CCDF of the received power $P_{r}$, we first obtain the Laplace transform of $P_{r}$ so that the CCDF can be obtained by inverse Laplace transform. The Laplace transform of $P_{r}$ is given by

$$
\begin{aligned}
\mathcal{L}_{P_{r}}(s) & =\mathbb{E}\left[\exp \left(-s P_{r}\right)\right] \\
& \stackrel{(a)}{=} \mathbb{E}_{\psi}\left[\prod_{x_{i} \in \psi} \mathbb{E}_{h_{x_{i}}}\left[\exp \left(-s a P h_{x_{i}}\left\|x_{i}\right\|^{-\alpha}\right)\right]\right] \\
& \stackrel{(b)}{=} \mathbb{E}_{\psi}\left[\prod_{x_{i} \in \psi} \frac{1}{1+s a P\left\|x_{i}\right\|^{-\alpha}}\right] \\
& \stackrel{(c)}{=} \exp \left[-2 \pi \lambda \delta \int_{0}^{\infty} \frac{v}{1+\frac{1}{s a P} v^{\alpha}} \mathrm{dv}\right]
\end{aligned}
$$

where $(a)$ follows from the independent and identically distributed (iid.) distribution of $h_{x}$ and its independence from the point process $\psi$, (b) follows from the use of the moment generating function of exponentially-distributed random variables, and (c) follows from the probability generating functional (PGFL) of the PPP, which states for some function $f(x)$ that $\mathbb{E}\left[\prod_{x \in \Phi} f(x)\right]=\exp \left(-\lambda \int_{\mathbb{R}^{2}}(1-f(x)) \mathrm{dx}\right)$. After some mathematical manipulations, we obtain

$$
\mathcal{L}_{P_{r}}(s)=\exp \left\{-\frac{2 \pi^{2} \lambda \delta}{\alpha \sin \left(\frac{2 \pi}{\alpha}\right)}(a P)^{\frac{2}{\alpha}} s^{\frac{2}{\alpha}}\right\} .
$$

In order to obtain the CCDF of $P_{r}$, the inverse Laplace transform of $\mathcal{L}_{P_{r}}(s)$ is required. We use the complex inversion integral formula for Laplace transforms with $s=0$ as a branch point of the integrand and the proper Bromwich contour that does not contain this branch point [21]. Note that similar results can be obtained using the Euler characterization [42].

\section{B. Proof of Lemma 1}

The probability that the near set $\Phi_{T}$ is empty can be derived as:

$$
\begin{aligned}
\mathbb{P}\left(\Phi_{T}=\emptyset\right) & =\mathbb{E}\left[\prod_{x_{i} \in \psi \backslash b_{0}} \mathbf{1}\left(a P h_{x_{i}}\left\|x_{i}\right\|^{-\alpha}<T\right)\right] \\
& \stackrel{(a)}{=} \mathbb{E}\left[\prod_{x_{i} \in \psi \backslash b_{0}} \mathbb{E}_{h_{x_{i}}} \mathbf{1}\left(a P h_{x_{i}}\left\|x_{i}\right\|^{-\alpha}<T\right)\right] \\
& \stackrel{(b)}{=} \mathbb{E}\left[\prod_{x_{i} \in \psi \backslash b_{0}} 1-e^{-\frac{1}{P_{n}}\left\|x_{i}\right\|^{\alpha}}\right] \\
& \stackrel{(c)}{=} \exp \left(-2 \pi \lambda \delta \int_{r}^{\infty} e^{-\frac{v^{\alpha}}{P_{n}}} v d \mathrm{v}\right) \\
& =\exp \left\{-\pi \lambda \delta\left[-r^{2} e^{-\frac{r^{\alpha}}{P_{n}}}+P_{n}^{\frac{2}{\alpha}} \Gamma\left(1+\frac{2}{\alpha}, \frac{r^{\alpha}}{P_{n}}\right)\right]\right\}
\end{aligned}
$$

where (a) follows from the iid. distribution of $h_{x}$ and its independence of the point process $\Phi_{\delta}$, (b) from the exponential distribution of $h_{x_{i}} \backsim \exp (1)$ and (c) from the probability generating functional of the PPP. The conditioned lower bound $P_{f_{l}^{r}}$ is then the complement of $\mathbb{P}\left(\Phi_{T}=\emptyset\right)$.

\section{Proof of Lemma 2}

To characterize the conditioned upper bound of the false wake-up probability we need first to evaluate $\mathbb{P}\left(I \geq T \mid I_{\Phi_{T}} \leq\right.$ $T)$ by using the Markov inequality as follows:

$$
\begin{aligned}
\mathbb{P}\left(I \geq T \mid I_{\Phi_{T}} \leq T\right) & =\mathbb{P}\left(I \geq T \mid \Phi_{T}=\emptyset\right) \\
& \stackrel{(a)}{\leq} \frac{\mathbb{E}\left[I \geq T \mid \Phi_{T}=\emptyset\right]}{T} \\
& =\frac{1}{T} \mathbb{E}\left[\sum_{x_{i} \in \psi \backslash b_{0}} a P h_{x_{i}}\left\|x_{i}\right\|^{-\alpha} \mathbf{1}\left(a P h_{x_{i}}\left\|x_{i}\right\|^{-\alpha}<T\right)\right] \\
& \stackrel{(b)}{=} \frac{2 \pi \lambda \delta}{T} \int_{r}^{\infty} a P v^{-\alpha} \mathbb{E}\left[h \mathbf{1}\left(a P h v^{-\alpha}<T\right)\right] v \mathrm{dv} \\
& =\frac{2 \pi \lambda \delta}{y} \int_{r}^{\infty} a P v^{-\alpha} \int_{0}^{\frac{T v^{\alpha}}{a P}} h e^{-h} \mathrm{dh} v \mathrm{dv},
\end{aligned}
$$

where (a) follows from the Markov inequality and (b) from the Campbell-Mecke theorem [39]. The conditioned upper bound can then be derived using 23 .

\section{Proof of Theorem 2}

The Laplace transform of the aggregate interference received at the tagged BS is obtained as:

$$
\begin{aligned}
\mathcal{L}_{I}(s) & =\mathbb{E}\left[e^{-s I}\right]=\mathbb{E}_{\tilde{\psi}}\left[e^{-s \sum_{y_{i} \in \tilde{\psi} \backslash y_{0}} \mathbf{1}\left(\gamma_{i}\left\|y_{i}\right\|^{-\alpha}<\rho\right) \gamma_{i} h_{y_{i}}\left\|y_{i}\right\|^{-\alpha}}\right] \\
& \stackrel{(a)}{=} \mathbb{E}_{\tilde{\psi}}\left[\prod_{y_{i} \in \tilde{\psi} \backslash y_{0}} \mathbb{E}_{\gamma_{i}, h_{y_{i}}}\left[e^{-s \mathbf{1}\left(\left\|y_{i}\right\|>\left(\frac{\gamma_{i}}{\rho}\right)^{\frac{1}{\alpha}}\right) \gamma_{i} h_{y_{i}}\left\|y_{i}\right\|^{-\alpha}}\right]\right] \\
& \stackrel{(b)}{=} \exp \left\{-2 \pi P_{w} \lambda \int_{\left(\frac{\gamma}{\rho}\right)^{\frac{1}{\alpha}}}^{\infty} \mathbb{E}_{\gamma, h}\left[\left(1-e^{-s \gamma h x^{-\alpha}}\right)\right] x \mathrm{dx}\right\} \\
& \stackrel{(c)}{=} \exp \left\{-2 \pi P_{w} \lambda \int_{\left(\frac{\gamma}{\rho}\right)^{\frac{1}{\alpha}}}^{\infty} \mathbb{E}_{\gamma}\left[\left(1-\frac{1}{1+s \gamma x^{-\alpha}}\right)\right] x \mathrm{dx}\right\} \\
& \stackrel{(d)}{=} \exp \left\{-2 \pi P_{w} \lambda s^{\frac{2}{\alpha}} \mathbb{E}\left[\gamma^{\frac{2}{\alpha}}\right] \int_{\frac{1}{(s \rho)}}^{\infty \frac{1}{\alpha}} \frac{u}{1+u^{\alpha}} \mathrm{du}\right\},
\end{aligned}
$$

where (a) follows from the independence between $\tilde{\psi}, \gamma^{i}$, and $h_{y_{i}}$ since $\left\{\gamma_{i}\right\}$ are iid.; (b) follows from the probability generation functional (PGFL) of the PPP of the interfering devices locations; (c) follows from the distribution of $h \backsim \exp (1)$ and (d) is obtained by changing the variable $u=\frac{1}{(s \gamma)^{\frac{1}{\alpha}}} x$. Note that the $\frac{2}{\alpha}$-moment of $\gamma$ can be obtained from Lemma 1 as $\mathbb{E}\left[\gamma^{\frac{2}{\alpha}}\right]=\frac{\rho^{\frac{2}{\alpha}}}{\pi \lambda}$. By plugging 50 and substituting $s=\frac{\tau}{\rho}$ in 37 the theorem is obtained.

\section{ACKNOWLEDGMENTS}

This work was made possible by a CNRS-L/AUB PhD Scholarship from the National Council for Scientific Research of the Lebanese Republic (CNRS-L) and the American University of Beirut (AUB). The statements made herein are solely the responsibility of the authors. 


\section{REFERENCES}

[1] S. Carson and P. Jonsson, "Ericsson mobility report: One the pulse of the networked society," tech. rep., 2015. Available online: http://goo.gl/5nSiwt.

[2] H. S. Dhillon, H. C. Huang, H. Viswanathan, and R. A. Valenzuela, "Power-efficient system design for cellular-based machine-to-machine communications," IEEE Trans. on Wireless Communications, vol. 12, pp. 5740-5753, Nov 2013.

[3] H. S. Dhillon, H. Huang, H. Viswanathan, and R. A. Valenzuela, "Fundamentals of throughput maximization with random arrivals for M2M communications," IEEE Transactions on Communications, vol. 62, pp. 4094-4109, Nov. 2014.

[4] G. Zhang, A. Li, K. Yang, L. Zhao, Y. Du, and D. Cheng, "Energyefficient power and time-slot allocation for cellular-enabled machine type communications," IEEE Communications Letters, vol. 20, pp. 368-371, Feb. 2016.

[5] A. Aijaz, M. Tshangini, M. Nakhai, X. Chu, and A.-H. Aghvami, "Energy-efficient uplink resource allocation in LTE networks with M2M/H2H co-existence under statistical QoS guarantees," IEEE Transactions on Communications, vol. 62, pp. 2353-2365, July 2014.

[6] H. Chao, Y. Chen, and J. Wu, "Power saving for machine to machine communications in cellular networks," in 2011 IEEE GLOBECOM Workshops (GC Wkshps), pp. 389-393, Dec. 2011.

[7] T. Tirronen, A. Larmo, J. Sachs, B. Lindoff, and N. Wiberg, "Machineto-machine communication with long-term evolution with reduced device energy consumption," Transactions on Emerging Telecommunications Technologies, vol. 24, no. 4, pp. 413-426, 2013.

[8] S. Jha, A. Koc, and R. Vannithamby, "Device power saving mechanisms for low cost MTC over LTE networks," in 2014 IEEE International Conference on Communications Workshops (ICC), pp. 412-417, June 2014.

[9] C. Rago, P. Willett, and Y. Bar-Shalom, "Censoring sensors: a lowcommunication-rate scheme for distributed detection," IEEE Transactions on Aerospace and Electronic Systems, vol. 32, pp. 554-568, Apr 1996.

[10] D. Ciuonzo, G. Romano, and P. S. Rossi, "Optimality of received energy in decision fusion over rayleigh fading diversity mac with non-identical sensors," IEEE Transactions on Signal Processing, vol. 61, pp. 22-27, Jan 2013.

[11] P. S. Rossi, D. Ciuonzo, K. Kansanen, and T. Ekman, "Performance analysis of energy detection for mimo decision fusion in wireless sensor networks over arbitrary fading channels," IEEE Transactions on Wireless Communications, vol. 15, pp. 7794-7806, Nov 2016.

[12] S. Wu, Y. Chen, K. Chai, F. Vazquez-Gallego, and J. Alonso-Zarate, "Analysis and performance evaluation of dynamic frame SlottedALOHA in wireless machine-to-machine networks with energy harvesting," in 2014 Globecom Workshops (GC Wkshps), pp. 1081-1086, Dec. 2014.

[13] M. D. Prete, D. Masotti, A. Costanzo, M. Magno, and L. Benini, "A 2.4 GHz-868 MHz dual-band wake-up radio for wireless sensor network and IoT," in 2015 IEEE 11th International Conference on Wireless and Mobile Computing, Networking and Communications (WiMob), pp. 322 328, Oct. 2015.

[14] P. Kolios, G. Ellinas, C. Panayiotou, and M. Polycarpou, "Energy efficient event-based networking for the internet of things," in 2016 IEEE 3rd World Forum on Internet of Things (WF-IoT), pp. 1-6, Dec. 2016.

[15] M. A. Kishk and H. S. Dhillon, "Downlink performance analysis of cellular-based IoT network with energy harvesting receivers," in 2016 IEEE Global Communications Conference, (USA, Washington DC), pp. 1-6, Dec. 2016.

[16] N. E. Roberts and D. D. Wentzloff, Ultra-Low Power Wake-Up Radios, pp. 137-162. Cham, Switzerland: Springer International Publishing, 2015.

[17] M. Park, S. Azizi, R. Stacey, and E. Perahia, "LP-WUR (low-power wake-up receiver): Enabling low power and low-latency capability for 802.11," Tech. Rep. IEEE 802.11-160027r0, 2016.

[18] N. Mazloum and O. Edfors, "Performance analysis and energy optimization of wake-up receiver schemes for wireless low-power applications," IEEE Transactions on Wireless Communications, vol. 13, pp. 70507061, Dec. 2014.

[19] T. Yoshiwaka, H. Yomo, and T. Ito, "Wake-up channel selection for on-demand WiFi wake-up using WLAN signals," in 2014 IEEE 79th Vehicular Technology Conference (VTC Spring), pp. 1-5, May 2014.

[20] C. Zhong, X. Chen, Z. Zhang, and G. K. Karagiannidis, "Wirelesspowered communications: Performance analysis and optimization,"
IEEE Transactions on Communications, vol. 63, pp. 5178-5190, Dec 2015.

[21] A. Sakr and E. Hossain, "Analysis of k-tier uplink cellular networks with ambient RF energy harvesting," IEEE Journal on Selected Areas in Communications, vol. 33, pp. 2226-2238, Oct 2015.

[22] I. Flint, X. Lu, N. Privault, D. Niyato, and P. Wang, "Performance analysis of ambient RF energy harvesting with repulsive point process modeling," IEEE Transactions on Wireless Communications, vol. 14 pp. 5402-5416, Oct 2015.

[23] S. Zhang, N. Zhang, S. Zhou, J. Gong, Z. Niu, and X. Shen, "Energyaware traffic offloading for green heterogeneous networks," IEEE Journal on Selected Areas in Communications, vol. 34, pp. 1116-1129, May 2016.

[24] X. Zhou, R. Zhang, and C. K. Ho, "Wireless information and power transfer: Architecture design and rate-energy tradeoff," IEEE Transactions on Communications, vol. 61, pp. 4754-4767, Nov. 2013.

[25] I. Flint, X. Lu, N. Privault, D. Niyato, and P. Wang, "Performance analysis of ambient RF energy harvesting: A stochastic geometry approach," in 2014 IEEE Global Communications Conference, pp. 1448-1453, Dec. 2014.

[26] X. Lu, I. Flint, D. Niyato, N. Privault, and P. Wang, "Performance analysis of simultaneous wireless information and power transfer with ambient RF energy harvesting," in 2015 IEEE Wireless Communications and Networking Conference (WCNC), pp. 1303-1308, Mar. 2015.

[27] I. Krikidis, "Simultaneous information and energy transfer in large-scale networks with/without relaying," IEEE Transactions on Communications, vol. 62, pp. 900-912, Mar. 2014.

[28] S. Zhou, T. Chen, W. Chen, and Z. Niu, "Outage minimization for a fading wireless link with energy harvesting transmitter and receiver," IEEE Journal on Selected Areas in Communications, vol. 33, pp. 496511, Mar. 2015.

[29] M. D. Renzo and W. Lu, "System-level analysis/optimization of cellular networks with simultaneous wireless information and power transfer: Stochastic geometry modeling," IEEE Transactions on Vehicular Technology, vol. 66, pp. 2251-2275, Mar. 2017.

[30] N. Kouzayha, Z. Dawy, and J. G. Andrews, "Analysis of a power efficient wake-up solution for M2M over cellular using stochastic geometry," in IEEE Global Conference on Communications, (USA, Washington DC), pp. 1-7, Dec. 2016

[31] 3GPP, "Cellular system support for ultra low complexity and low throughput internet of things, (release 13)," Technical Report TR 45.820, 2015.

[32] 3GPP, "Evolved universal terrestrial radio access (E-UTRA); Radio resource control (RRC)," Protocol specification (version 10.4.0) TS $36.331,2011$

[33] M. Haenggi, "The mean interference-to-signal ratio and its key role in cellular and amorphous networks," IEEE Wireless Communications Letters, vol. 3, pp. 597-600, Dec 2014.

[34] A. Guo and M. Haenggi, "Spatial stochastic models and metrics for the structure of base stations in cellular networks," IEEE Transactions on Wireless Communications, vol. 12, pp. 5800-5812, Nov 2013.

[35] R. K. Ganti and M. Haenggi, "Asymptotics and approximation of the sir distribution in general cellular networks," IEEE Transactions on Wireless Communications, vol. 15, pp. 2130-2143, Mar 2016.

[36] H. ElSawy, A. Sultan-Salem, M. S. Alouini, and M. Z. Win, "Modeling and analysis of cellular networks using stochastic geometry: A tutorial," IEEE Communications Surveys Tutorials, vol. 19, pp. 167-203, Firstquarter 2017.

[37] H. ElSawy, E. Hossain, and M. Haenggi, "Stochastic geometry for modeling, analysis, and design of multi-tier and cognitive cellular wireless networks: A survey," IEEE Communications Surveys Tutorials, vol. 15, pp. 996-1019, Thirdquarter 2013.

[38] H. ElSawy and M. S. Alouini, "On the meta distribution of coverage probability in uplink cellular networks," IEEE Communications Letters, vol. 21 , pp. $1625-1628$, July 2017.

[39] S. Chiu, D. Stoyan, W. Kendall, and J. Mecke, Stochastic Geometry and Its Applications. Wiley Series in Probability and Statistics, Wiley, 2013.

[40] J. G. Andrews, F. Baccelli, and R. K. Ganti, "A tractable approach to coverage and rate in cellular networks," IEEE Transactions on Communications, vol. 59, pp. 3122-3134, Nov. 2011.

[41] A. Sakr and E. Hossain, "Location-aware cross-tier coordinated multipoint transmission in two-tier cellular networks," IEEE Transactions on Wireless Communications, vol. 13, pp. 6311-6325, Nov. 2014.

[42] J. Abate and W. Whitt, "Numerical inversion of laplace transforms of probability distributions," ORSA Journal on Computing, vol. 7, pp. 3643, Feb. 1995 
[43] S. Weber, J. G. Andrews, and N. Jindal, "An overview of the transmission capacity of wireless networks," IEEE Transactions on Communications, vol. 58, pp. 3593-3604, Dec. 2010.

[44] H. S. Dhillon, R. K. Ganti, and J. G. Andrews, "Load-aware modeling and analysis of heterogeneous cellular networks," IEEE Transactions on Wireless Communications, vol. 12, pp. 1666-1677, Apr. 2013.

[45] H. ElSawy and E. Hossain, "On stochastic geometry modeling of cellular uplink transmission with truncated channel inversion power control," IEEE Transactions on Wireless Communications, vol. 13, pp. 44544469, Aug. 2014

[46] T. D. Novlan, H. S. Dhillon, and J. G. Andrews, "Analytical modeling of uplink cellular networks," IEEE Transactions on Wireless Communications, vol. 12, pp. 2669-2679, June 2013.

[47] M. D. Renzo and P. Guan, "Stochastic geometry modeling and systemlevel analysis of uplink heterogeneous cellular networks with multiantenna base stations," IEEE Transactions on Communications, vol. 64 pp. 2453-2476, June 2016.

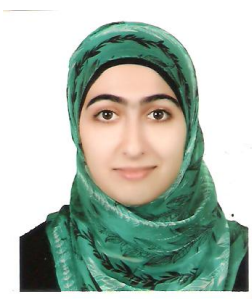

Nour Kouzayha received her M.Sc. degree in telecommunication system technologies and her M.E. degree in computer and communications engineering in 2013 from the Lebanese University, Beirut, Lebanon. Currently, she is a PhD candidate in the Department of Electrical and Computer Engineering at the American University of Beirut, Beirut, Lebanon. Her research interests are in the area of communication theory and networks, cellular technologies, RF energy harvesting, Internet of things applications and stochastic geometry.

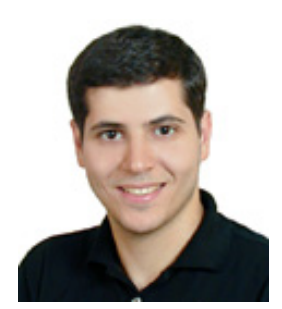

Zaher Dawy (SM'09) received the B.E. degree in computer and communications engineering from the American University of Beirut (AUB), Beirut, Lebanon, in 1998 and the M.E. and Dr.-Ing. degrees in communications engineering from Munich University of Technology (TUM), Munich, Germany, in 2000 and 2004, respectively. Since September 2004, he has been with AUB, where he is currently a Professor of Electrical and Computer Engineering. His research and teaching interests include distributed and cooperative communications, cellular technologies, context-aware mobile computing, computational biology, and biomedical engineering. Dr. Dawy currently serves on the editorial board for IEEE Transactions on Communications, IEEE Transactions on Wireless Communications, IEEE Communications Surveys and Tutorials, and Elsevier Physical Communications. Before, he served as an Executive Editor for Wiley Transactions on Emerging Telecommunications Technologies and as the Chair of the IEEE Communications Society Lebanon Chapter. He is the recipient of the Abdul Hameed Shoman Award for Young Arab Researchers in 2012, IEEE Communications Society 2011 Outstanding Young Researcher Award in Europe, Middle East, and Africa Region, AUB Teaching Excellence Award in 2008, Best MSCE Graduate Award from TUM in 2000, Youth and Knowledge Siemens Scholarship for Distinguished Students in 1999, and Distinguished Graduate Medal of Excellence from Hariri Foundation in 1998.

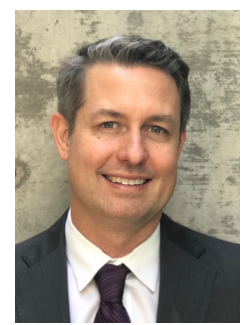

Jeffrey Andrews (S'98, M'02, SM'06, F'13) received the B.S. in Engineering with High Distinction from Harvey Mudd College, and the M.S. and Ph.D. in Electrical Engineering from Stanford University. $\mathrm{He}$ is the Cullen Trust Endowed Professor (\#1) of ECE at the University of Texas at Austin. He developed Code Division Multiple Access systems at Qualcomm from 1995-97, and has consulted for entities including Apple, Samsung, Version, AT\&T, the WiMAX Forum, Intel, Microsoft, Clearwire, Sprint, and NASA. He is a member of the Technical Advisory Board of Artemis Networks and GenXComm, and is co-author of the books Fundamentals of WiMAX (Prentice-Hall, 2007) and Fundamentals of LTE (Prentice-Hall, 2010). He was the Editor-in-Chief of the IEEE Transactions on Wireless Communications from 2014-2016 and is Chair of the IEEE Communications Society Emerging Technologies Committee.

Dr. Andrews is an ISI Highly Cited Researcher and has been co-recipient of fourteen best paper awards including the 2016 IEEE Communications Society \& Information Theory Society Joint Paper Award, the 2014 IEEE Stephen O. Rice Prize, and the 2014 IEEE Leonard G. Abraham Prize, the 2011 and 2016 IEEE Heinrich Hertz Prize, and the 2010 IEEE ComSoc Best Tutorial Paper Award. He received the 2015 Terman Award, the NSF CAREER Award, is an IEEE Fellow, and is an elected member of the Board of Governors of the IEEE Information Theory Society.

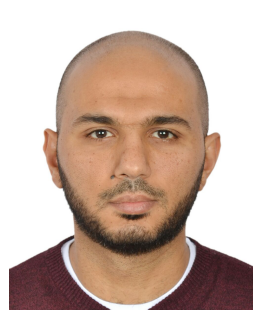

Hesham ElSawy (S'10, M'14, SM'17) received the B.Sc. degree in Electrical Engineering from Assiut University, Assiut, Egypt, in 2006, the M.Sc. degree in Electrical Engineering from Arab Academy for Science and Technology, Cairo, Egypt, in 2009, and the Ph.D. degree in Electrical Engineering from the University of Manitoba, Winnipeg, MB Canada, in 2014. Currently, he is a postdoctoral fellow with the Computer, Electrical, and Mathematical Sciences and Engineering Division, King Abdullah University of Science and Technology (KAUST), Saudi Arabia, and also has an adjunct affiliation to the school of Computer Science \& Engineering, York University, Canada. During the period of 2006-2010, he worked at the National Telecommunication Institute, Egypt, where he conducted professional training both at the national and international levels, as well as research on network planning. From 2010 to 2014, he worked with TRTech, Winnipeg, MB, Canada, as a Student Researcher. He has received several academic awards, including the NSERC Industrial Postgraduate Scholarship during the period of $2010-2013$, and the TRTech Graduate Students Fellowship in the period of $2010-2014$. He also received the IEEE communications society best survey paper award in 2017, the best scientific contribution award to the ISWCS 2017, and the best paper award at the ICC workshop on small cells and 5G networks in 2015. He is recognized as an exemplary reviewer by the IEEE Transactions on Communications for the three consecutive years 2015-2017. His research interests include statistical modeling of wireless networks, stochastic geometry, and queuing analysis for wireless communication networks. 